\title{
Structure, diffusion and rheology of Brownian suspensions by Stokesian Dynamics simulation
}

\author{
By DAVID R. FOSS AND JOHN F. BRADY \\ Division of Chemistry and Chemical Engineering, California Institute of Technology, Pasadena, \\ CA 91125, USA
}

(Received 20 April 1999 and in revised form 25 September 1999)

The non-equilibrium behaviour of concentrated colloidal dispersions is studied using Stokesian Dynamics, a molecular-dynamics-like simulation technique for analysing suspensions of particles immersed in a Newtonian fluid. The simulations are of a monodisperse suspension of Brownian hard spheres in simple shear flow as a function of the Péclet number, $P e$, which measures the relative importance of hydrodynamic and Brownian forces, over a range of volume fraction $0.316 \leqslant \phi \leqslant 0.49$. For $P e<10$, Brownian motion dominates the behaviour, the suspension remains well-dispersed, and the viscosity shear thins. The first normal stress difference is positive and the second negative. At higher $P e$, hydrodynamics dominate resulting in an increase in the long-time self-diffusivity and the viscosity. The first normal stress difference changes sign when hydrodynamics dominate. Simulation results are shown to agree well with both theory and experiment.

\section{Introduction}

Suspensions of small particles dispersed in a fluid occur in a variety of natural and industrial settings, such as slurries, paints, pastes, many foodstuffs, and ceramic sols. In these microstructured fluids the suspended particles interact through hydrodynamic, interparticle, and Brownian (or thermal) forces. The balance between thermal and interparticle forces determines the equilibrium behaviour. Under the action of an external driving force such as shear, hydrodynamic forces come into play and compete with thermal and interparticle forces to set the structure and determine properties. There have been a number of experiments on well-characterized model hard-sphere systems (de Kruif et al. 1985; van der Werff \& de Kruif 1989; van der Werff et al. 1989; Ackerson 1990; etc.) that have greatly advanced our understanding of colloidal dispersions, and, along with scaling theories for the behaviour at high solids concentration (Brady 1993b; Brady \& Morris 1997) and Stokesian Dynamics simulations (Bossis \& Brady 1984, 1987, 1989; Brady \& Bossis 1985, 1988; Phung \& Brady 1992; Phung 1993; Phung, Brady \& Bossis 1996; Ball \& Melrose 1995; Dratler \& Schowalter 1996) a complete picture is emerging.

In this work we report on Stokesian Dynamics simulation studies of rheology, diffusion, and structure of concentrated monodisperse suspensions of hard spheres. Stokesian Dynamics is a general molecular-dynamics-like method for simulating suspensions at low particle Reynolds number that accurately calculates the manybody interactions necessary to capture the hydrodynamic forces transmitted through the fluid. In a hard-sphere suspension particles interact through hydrodynamic and 
Brownian forces only, and the system is described by the minimal number of parameters - the volume fraction $\phi$ and the Péclet number, $P e$. The Péclet number is the ratio of hydrodynamic shear to thermal forces, or alternatively the ratio of Brownian and flow time scales and is given by $P e=\dot{\gamma} a^{2} / D$, where $\dot{\gamma}$ is the magnitude of the shear rate, and $D=k T / 6 \pi \eta a$ is the Stokes-Einstein diffusivity of an isolated spherical particle of radius $a$ and thermal energy $k T$ in a fluid of viscosity $\eta$.

As the Péclet number is varied the simulations reveal two characteristic regimes of suspension behaviour. At low Péclet number $(P e \leqslant 10)$ the equilibrium structure is distorted by the flow and the suspension viscosity shear thins. The shear thinning is caused by the decrease of the direct Brownian contribution to the stress as the magnitude of the structural deformation does not match the flow strength. The hydrodynamic contribution to the stress remains constant and equal to the high-frequency dynamic viscosity, $\eta_{\infty}^{\prime}$, throughout the shear thinning process. The zero-shear-rate viscosity is determined both as the limiting value of the steady shear viscosity as the shear rate vanishes and from the decay of the shear stress autocorrelation at equilibrium (Nägele \& Bergenholtz 1998). The simulation viscosities show no variation with the size of, or the number of, particles in a unit cell $(27 \leqslant N \leqslant 123)$ and compare well with experiment (van der Werff \& de Kruif 1989; D'Haene, Mewis \& Fuller 1993). Normal stress differences have proven difficult to measure accurately at low Péclet number but are determined by the direct Brownian contribution to the stress. The first normal stress difference is positive and the second negative. Unlike polymer systems, however, both normal stress differences are of comparable magnitude.

At high Péclet number the shearing forces overcome Brownian motion and push particles into close contact where the short-range hydrodynamic lubrication forces are important. The suspension exhibits shear thickening due to the increase in the hydrodynamic contribution to the stress caused by the formation of clusters that are bound by lubrication forces as first shown by Bossis \& Brady (1989). The first normal stress difference changes sign, and both normal stress differences are negative and appear to approach an $O(\eta \dot{\gamma})$ high- $P e$ asymptote. The long-time self-diffusivity also grows dramatically with $P e$ and reaches a purely hydrodynamic $O\left(\dot{\gamma} a^{2}\right)$ limit at high $P e$. The appearance of diffusive motion and normal stress differences in the deterministic pure hydrodynamic limit is surprising. Recent theoretical work by Brady \& Morris (1997) has shown, however, that the high-Pe limit is singular, and the residual effect of weak Brownian motion introduces irreversibility, which may result in finite normal stress differences and diffusive motion.

An unfortunate error in the simulation code used in our previous work (Phung \& Brady 1992; Phung 1993; Phung et al. 1996) has been discovered and corrected for this paper (see $\S 2$ for details of this error). The main effect of this correction is the absence of a flow-induced string-ordered phase at intermediate Péclet numbers and high concentrations that was observed in the earlier work. The low-Péclet-number shearthinning regime $(P e \leqslant 1)$ is not affected. The high-Péclet-number shear-thickening behaviour is changed (slightly) quantitatively, but not qualitatively. Thus this paper has a dual purpose: to correct the earlier results of Phung et al. (1996) which were for a single volume fraction $\phi=0.45$; and to present results for a wide range of volume fractions $(0.316 \leqslant \phi \leqslant 0.49)$ and to make comparison with theories for shear thinning and thickening that have been developed recently.

In the next section we outline the Stokesian Dynamics simulation method. In $\S 3$ we present and discuss the simulation results for rheology, diffusion, and structure in concentrated colloidal dispersions of Brownian hard spheres. Conclusions are given in $\S 4$. 


\section{Simulation method}

A detailed derivation of the simulation method has appeared in the literature (Durlofsky, Brady \& Bossis 1987; Bossis \& Brady 1987, 1989; Brady \& Bossis 1988; Brady et al. 1988; Phung et al. 1996), so we shall proceed quickly. For $N$ rigid particles suspended in an incompressible Newtonian fluid of viscosity $\eta$ and density $\rho$, the fluid motion is governed by the Navier-Stokes equations, while the particle motion is described by the coupled $N$-body Langevin equation:

$$
\boldsymbol{m} \cdot \frac{\mathrm{d} \boldsymbol{U}}{\mathrm{d} t}=\boldsymbol{F}^{H}+\boldsymbol{F}^{P}+\boldsymbol{F}^{B} .
$$

In (1) $\boldsymbol{m}$ is the generalized mass/moment of inertia tensor, $\boldsymbol{U}$ is the particle translational/rotational velocity vector of dimension $6 N$, and the $6 N$ force/torque vectors $\boldsymbol{F}$ represent: $(a)$ the hydrodynamic forces $\boldsymbol{F}^{H}$ exerted on the particles due to their motion relative to the fluid, $(b)$ the deterministic non-hydrodynamic forces $\boldsymbol{F}^{P}$, which may be either interparticle or external, and $(c)$ the stochastic forces $\boldsymbol{F}^{B}$ that give rise to Brownian motion.

When the particle Reynolds number is small, i.e. $R e=\rho a^{2} \dot{\gamma} / \eta \ll 1$ for the shear flows considered here, the hydrodynamic force/torque exerted on the particles in a suspension undergoing a bulk linear flow is

$$
\boldsymbol{F}^{H}=-\boldsymbol{R}_{F U} \cdot(\boldsymbol{U}-\langle\boldsymbol{U}\rangle)+\boldsymbol{R}_{F E}:\langle\boldsymbol{E}\rangle .
$$

In (2), $\langle\boldsymbol{U}\rangle=\langle\dot{\boldsymbol{\Gamma}}\rangle \cdot \boldsymbol{x}$ is the imposed bulk flow evaluated at the particle centres, $\langle\dot{\boldsymbol{\Gamma}}\rangle=\langle\boldsymbol{E}\rangle+\langle\boldsymbol{\Omega}\rangle$, and $\langle\boldsymbol{E}\rangle$ and $\langle\boldsymbol{\Omega}\rangle$ are the bulk rate-of-strain and vorticity tensors, respectively, and are constant in space but may be arbitrary functions of time. The configuration-dependent resistance tensors $\boldsymbol{R}_{F U}(\boldsymbol{x})$ and $\boldsymbol{R}_{F E}(\boldsymbol{x})$ give the hydrodynamic force/torque on the particles due to their motion relative to the fluid and due to an imposed flow, respectively. The vector $\boldsymbol{x}$ represents the generalized configuration vector specifying the location and orientation of all $N$ particles.

The deterministic, non-hydrodynamic force $\boldsymbol{F}^{P}$ is arbitrary. The stochastic or Brownian force $\boldsymbol{F}^{B}$ arises from the thermal fluctuations in the fluid and is characterized by

$$
\overline{\boldsymbol{F}^{B}}=0 \quad \text { and } \quad \overline{\boldsymbol{F}^{B}(0) \boldsymbol{F}^{B}(t)}=2 k T \boldsymbol{R}_{F U} \delta(t) .
$$

In (3) the overbar denotes an average over the rapid fluctuations of the solvent molecules, $k$ is Boltzmann's constant, $T$ is the absolute temperature, and $\delta(t)$ is the delta function. The amplitude of the correlation between the Brownian forces at time 0 and at time $t$ results from the fluctuation-dissipation theorem.

The evolution equation for the particles is obtained by integrating (1) over a time step $\Delta t$ that is large compared with $\tau_{p}$, the inertial relaxation time $\left(\tau_{p}=m / 6 \pi \eta a\right)$, but small compared with the time over which the configuration changes. A second integration in time produces the evolution equation for the particle positions (both translational and orientational) with error of $o(\Delta t)$ :

$$
\left.\begin{array}{c}
\Delta \boldsymbol{x}=P e\left\{\langle\boldsymbol{U}\rangle+\boldsymbol{R}_{F U}^{-1} \cdot\left[\boldsymbol{R}_{F E}:\langle\boldsymbol{E}\rangle+\dot{\gamma}^{*-1} \boldsymbol{F}^{P}\right]\right\} \Delta t+\nabla \cdot \boldsymbol{R}_{F U}^{-1} \Delta t+\boldsymbol{X}(\Delta t), \\
\overline{\boldsymbol{X}}=0 \text { and } \overline{\boldsymbol{X}(\Delta t) \boldsymbol{X}(\Delta t)}=2 \boldsymbol{R}_{F U}^{-1} \Delta t .
\end{array}\right\}
$$

Here, $\Delta \boldsymbol{x}$ is the change in particle position during the time step $\Delta t$, and $\boldsymbol{X}(\Delta t)$ is a random displacement due to Brownian motion that has zero mean and covariance given by the inverse of the resistance tensor. In (4) $\boldsymbol{x}$ has been non-dimensionalized by the characteristic particle size $a$; the time by the diffusive time scale $a^{2} / D$, where 
$D=k T / 6 \pi \eta a$ is the diffusion coefficient of a single isolated particle; the rate-ofstrain tensor $\langle\boldsymbol{E}\rangle$ by $\dot{\gamma}$, where $\dot{\gamma}=|\langle\dot{\boldsymbol{\Gamma}}\rangle|$ is the magnitude of the shear rate; the shear forces by $6 \pi \eta a^{2} \dot{\gamma}$; and the interparticle forces by their magnitude $\left|\boldsymbol{F}^{P}\right|$. The Péclet number, $P e=\dot{\gamma} a^{2} / D=6 \pi \eta a^{3} \dot{\gamma} / k T$, measures the relative importance of shear and Brownian forces, and $\dot{\gamma}^{*}=6 \pi \eta a^{2} \dot{\gamma} /\left|\boldsymbol{F}^{P}\right|$ is a non-dimensional shear rate giving the relative importance of shear and interparticle or externally imposed forces. For simulations where $P e>1$ it is convenient to non-dimensionalize the time step $\Delta t$ with the inverse shear rate $1 / \dot{\gamma}$. This is done by replacing $\Delta t$ in (4) by $\Delta t / P e$ producing an alternative form of the evolution equation:

$$
\left.\begin{array}{c}
\Delta \boldsymbol{x}=\left\{\langle\boldsymbol{U}\rangle+\boldsymbol{R}_{F U}^{-1} \cdot\left[\boldsymbol{R}_{F E}:\langle\boldsymbol{E}\rangle+\dot{\gamma}^{*-1} \boldsymbol{F}^{P}\right]\right\} \Delta t+\frac{1}{P e} \nabla \cdot \boldsymbol{R}_{F U}^{-1} \Delta t+\frac{1}{P e^{1 / 2}} \boldsymbol{X}(\Delta t), \\
\overline{\boldsymbol{X}}=0 \text { and } \overline{\boldsymbol{X}(\Delta t) \boldsymbol{X}(\Delta t)}=2 \boldsymbol{R}_{F U}^{-1} \Delta t .
\end{array}\right\}
$$

The high-Péclet-number form of the evolution equation was incorrect in the Stokesian Dynamics code that was used for recently published results (Phung \& Brady 1992; Phung 1993 and Phung et al. 1996). The random displacement term, $\boldsymbol{X}(\Delta t)$, had a $1 / P e$ coefficient which is too small for $P e>1$ which affected the results of those simulations. If we had simple diffusive motion in which the mobility was independent of the configuration, then one could simply rescale the shear rate by $P e^{1 / 2}$ to convert the incorrect $(1 / P e)$ results to the correct behaviour $\left(1 / P e^{1 / 2}\right)$. However, the configuration-dependent mobility gives rise to the deterministic displacement $\nabla \cdot \boldsymbol{R}_{F U}^{-1} \Delta t$, which acts like a repulsive force between particles, and this rescaling produces too large a repulsive force $\left(1 / P e^{1 / 2}\right.$ instead of $\left.1 / P e\right)$. It is well known that suspensions with non-hydrodynamic repulsive forces order into string phases if the forces are strong enough and the volume fraction is high enough (Bossis \& Brady 1984; Rastogi 1995). Thus, the error in the earlier simulations acted to enhance this 'repulsive' force and produce ordering where there should be none as we show in this work. Note, this only affects the behaviour for $P e>1$; the correct scaling for $P e<1$ is used in Phung et al. (1996).

The macroscopic properties are found from appropriate definitions and averages over particles and over time in a dynamic simulation. Here we shall be primarily interested in diffusion and rheology. Several 'particle diffusivities' may be defined. The short-time self-diffusivity $\boldsymbol{D}_{0}^{s}$, which measures the average instantaneous mobility of a particle, is given by an average over all configurations: $\boldsymbol{D}_{0}^{s}=\left\langle\boldsymbol{D}_{i i}\right\rangle$, where the subscript $i i$ (no sum on $i$ ) indicates that only the diagonal or self terms are included in the sum, and the angle brackets denote an average over all configurations and all identical particles. The $N$-particle diffusion tensor $\boldsymbol{D}$ is given by the Stokes-Einstein relation:

$$
\boldsymbol{D} \equiv k T \boldsymbol{R}_{F U}^{-1} .
$$

The long-time self-diffusivity $\boldsymbol{D}_{\infty}^{s}$, which measures the ability of a particle to wander far from its starting point, is defined as the limit as time approaches infinity of one half of the time rate of change of the mean-square position of a particle:

$$
\boldsymbol{D}_{\infty}^{s}=\lim _{t \rightarrow \infty} \frac{1}{2} \frac{\mathrm{d}}{\mathrm{d} t}\left\langle(\boldsymbol{x}-\langle\boldsymbol{x}\rangle)^{2}\right\rangle .
$$

For rheology, the bulk stress $\langle\boldsymbol{\Sigma}\rangle$ is needed. This is defined as an average over the volume $V$ containing the $N$ particles and is given by

$$
\langle\boldsymbol{\Sigma}\rangle=-\langle p\rangle \boldsymbol{I}+2 \eta\langle\boldsymbol{E}\rangle+\left\langle\boldsymbol{\Sigma}_{p}\right\rangle,
$$


where $\langle p\rangle$ is a constant setting the level of the pressure in the incompressible medium, and $2 \eta\langle\boldsymbol{E}\rangle$ is the deviatoric stress contribution from the fluid. The particle contribution to the stress $\left\langle\boldsymbol{\Sigma}_{p}\right\rangle$ is given by

$$
\left\langle\boldsymbol{\Sigma}_{p}\right\rangle=-n k T \boldsymbol{I}+n\left\{\left\langle\boldsymbol{S}^{H}\right\rangle+\left\langle\boldsymbol{S}^{P}\right\rangle+\left\langle\boldsymbol{S}^{B}\right\rangle\right\} .
$$

Here, $-n k T I$ is the isotropic stress associated with the thermal energy of the Brownian particles, $\boldsymbol{I}$ is the isotropic tensor, and $n$ is the number density of particles. There are three contributions to the bulk stress: $(a)$ a mechanical or contact stress transmitted by the fluid due to the shear flow, $\left\langle\boldsymbol{S}^{H}\right\rangle ;(b)$ a stress due to the interparticle forces, $\left\langle\boldsymbol{S}^{P}\right\rangle$; and (c) a direct contribution from Brownian $\left\langle\boldsymbol{S}^{B}\right\rangle$; they are given by

$$
\begin{aligned}
& \left\langle\boldsymbol{S}^{H}\right\rangle=-\left\langle\boldsymbol{R}_{S U} \cdot \boldsymbol{R}_{F U}^{-1} \cdot \boldsymbol{R}_{F E}-\boldsymbol{R}_{S E}\right\rangle:\langle\boldsymbol{E}\rangle, \\
& \left\langle\boldsymbol{S}^{P}\right\rangle=-\left\langle\left(\boldsymbol{R}_{S U} \cdot \boldsymbol{R}_{F U}^{-1}+\boldsymbol{x} \boldsymbol{)}\right) \cdot \boldsymbol{F}^{P}\right\rangle, \\
& \left\langle\boldsymbol{S}^{B}\right\rangle=-k T\left\langle\nabla \cdot\left(\boldsymbol{R}_{S U} \cdot \boldsymbol{R}_{F U}^{-1}\right)\right\rangle .
\end{aligned}
$$

The configuration-dependent resistance tensors $\boldsymbol{R}_{S U}(\boldsymbol{x})$ and $\boldsymbol{R}_{S E}(\boldsymbol{x})$ are similar to $\boldsymbol{R}_{F U}$ and $\boldsymbol{R}_{F E}$ and relate the particle 'stresslet' $\boldsymbol{S}$ to the particle velocities and to the imposed rate of strain, respectively. The stresslet is the symmetric first moment of the force distribution integrated over the particle surface.

The hydrodynamic resistance tensors $\boldsymbol{R}_{F U}, \boldsymbol{R}_{F E}$, etc. that appear in the evolution equation and macroscopic stress are computed in the same manner as discussed in Phung et al. (1996) and are not repeated here. Suffice it to say that the method accurately accounts for the near-field lubrication effects and the dominant many-body interactions. Periodic boundary conditions are used and all long-range hydrodynamic interactions are accelerated with the Ewald summation technique. As currently implemented, calculation of the hydrodynamic interactions requires $O\left(N^{3}\right)$ operations and thus limits the simulations to small systems $(27 \leqslant N \leqslant 123)$. The same time integration scheme used by Phung et al. (1996) is employed here.

The simulation results are for a monodisperse suspension of Brownian hard spheres. For particles interacting as hard spheres the interparticle force is identically zero, $\boldsymbol{F}^{P} \equiv$ 0 . The no-slip hydrodynamic boundary condition at particle surfaces guarantees that the particles behave as hard spheres (Brady 1993a). This can be appreciated by noting that an interparticle force of hard-sphere type at contact $r=2 a$ between two particles, $\boldsymbol{F}^{P}=\frac{1}{2} k T \hat{r} \delta(r-2 a)$, has no dynamical consequence. Since the relative mobility of two particles vanishes at contact as $r-2 a$ due to the lubrication interactions, the relative velocity caused by a hard-sphere force is proportional to $(r-2 a) \delta(r-2 a)$ and vanishes. Simulations with $\boldsymbol{F}^{P} \equiv 0$ at $P e \equiv 0$ were shown by Phung et al. (1996) to produce precisely the expected hard-sphere behaviour. Similarly, the stress contribution from hard-sphere forces at contact is zero $\left(\boldsymbol{S}^{P} \equiv 0\right)$.

\section{Results}

A large number of simulations were performed for a range in volume fraction of $0.316 \leqslant \phi \leqslant 0.49$, Péclet numbers ranging from 0 to $10^{4}$ and the number of particles $N$ in the unit cell ranging from 27 to 123 . Many of the long runs were divided into statistically independent subintervals in order to determine the statistical variation in the properties. All runs for all Péclet numbers were started using hard-sphere equilibrium configurations obtained from a Monte Carlo procedure. For each run, the first 10000 to 20000 time steps were ignored when computing average properties. The properties reported are averages over all particles and over time. All simulations 
are for simple shear flow with the flow, velocity-gradient, and vorticity directions, respectively, along the three axes $(x, y, z)$ of the cubic unit cell. The presentation below is divided into three parts: rheology, diffusion and structure.

\subsection{Rheology}

\subsubsection{Shear viscosity}

In steady simple shear flow the viscosity of a suspension is related to the $(x, y)$ components of the bulk stress and rate of strain in the following manner:

$$
\eta_{r} \equiv \frac{\Sigma_{x y}}{2 \eta E_{x y}} .
$$

The individual Brownian $\left(\mathbf{S}^{B}\right)$ and hydrodynamic $\left(\mathbf{S}^{H}\right)$ contributions to the relative viscosity are denoted as $\eta_{r}^{B}$ and $\eta_{r}^{H}$, respectively; hence

$$
\eta_{r}=1+\eta_{r}^{B}+\eta_{r}^{H},
$$

where the 1 is the solvent contribution.

First, we can analyse the fluctuations in stress at equilibrium and extract the zeroshear limiting viscosity from the following dimensional Green-Kubo formula ( Nägele \& Bergenholtz 1998):

$$
\eta_{0}=\eta_{\infty}^{\prime}+\frac{V}{k T} \int_{0}^{\infty}\left\langle\sigma_{x y}(t) \sigma_{x y}(0)\right\rangle \mathrm{d} t .
$$

Here, $\eta_{\infty}^{\prime}$ is the high-frequency dynamic viscosity, which represents the viscous contribution to the stress at equilibrium, and is easily calculated from simulation. The instantaneous Brownian shear stress is given by $\sigma_{x y}(t)$. Although its average over long times is zero, the Brownian stress fluctuates along with the microstructure due to Brownian motion. The shear-stress autocorrelation function $\left\langle\sigma_{x y}(t) \sigma_{x y}(0)\right\rangle$ analyses the nature of the relaxation of these fluctuations. Here, we use the subscript $x y$ for simplicity, but since there are no preferred directions at equilibrium, we can also autocorrelate the $x z$ - and $y z$-components of the Brownian stress tensor and average the three functions to reduce statistical noise. Simulations were run at $P e=0$ using a time step of $\Delta t=5 \times 10^{-4}$ for 400000 steps to calculate the shear-stress autocorrelation function. A discussion of the time-dependent behaviour of the autocorrelation function is presented elsewhere (Foss 1999). Here we are only interested in the zero-shear viscosity $\eta_{0}$.

The zero-shear-rate viscosity from the Green-Kubo formula is shown in table 1 and compared with data from steady-shear simulation and experiment as a function of volume fraction, $\phi$, in figure 1 . The values calculated in this work from GreenKubo analysis are virtually indistinguishable from the previously reported viscosities of Phung (1993) determined from steady-state averages at the lowest shear rate $(P e=0.01)$. This gives us confidence that the simulations were indeed performed at low enough $P e$ to measure the limiting zero-shear viscosity. General agreement is found between the values obtained from Stokesian Dynamics and the experimental data, which are in the low-Pe limit.

We now turn our focus to the shear-rate dependence of the suspension stress. A representative viscosity versus Péclet number curve (figure 2) for a volume fraction of 0.45 and $N=27$ shows the individual contributions to the viscosity as a function of the Péclet number. The Brownian viscosity shear thins, becoming insignificant compared to the hydrodynamic viscosity for $P e>10$. The hydrodynamic viscosity 


\begin{tabular}{llll}
\multicolumn{1}{c}{$\phi$} & $\eta_{0}^{B}$ & $\eta_{\infty}^{\prime}$ & $\eta_{0}$ \\
0.20 & 0.14 & 1.81 & 1.95 \\
0.316 & 0.68 & 2.87 & 3.55 \\
0.37 & 2.0 & 3.66 & 5.7 \\
0.40 & 2.8 & 4.28 & 7.1 \\
0.419 & 4.8 & 4.78 & 9.6 \\
0.45 & 9.5 & 5.61 & 15.1 \\
0.47 & 18.8 & 6.19 & 24.0 \\
0.49 & 24.2 & 7.05 & 31.3
\end{tabular}

TABLE 1. Data for zero-shear viscosity, $\eta_{0}$, and its different contributions as a function of $\phi$ from Stokesian Dynamics, $N=27$. The Brownian contribution, $\eta_{0}^{B}$, is calculation from a Green-Kubo formula involving time-integration of the shear-stress autocorrelation function, equation (12). The hydrodynamic contribution is the high-frequency dynamic viscosity, $\eta_{\infty}^{\prime}$, and is determined by calculating $1+\eta^{H}$ in the $P e \rightarrow 0$ limit. All viscosities are non-dimensionalized by the solvent viscosity, $\eta$.

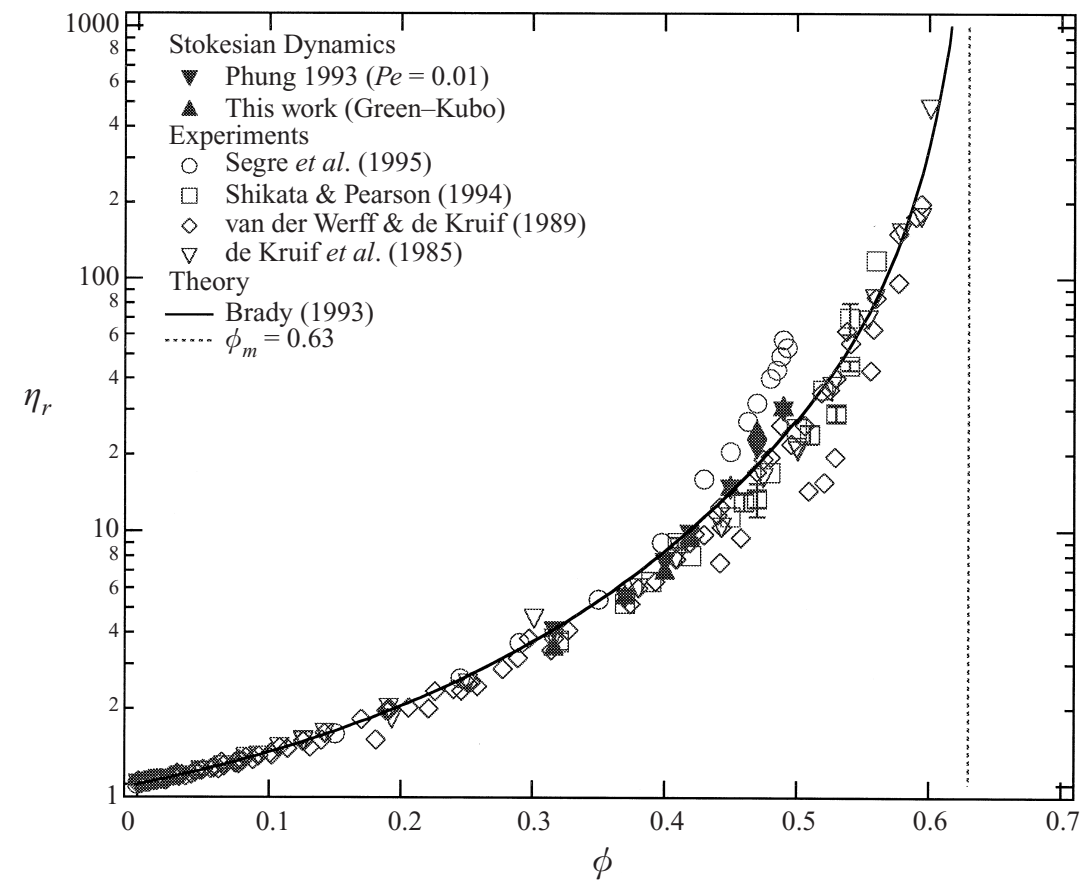

FIGURE 1. Zero-shear limiting relative viscosity, $\eta_{0}$, as a function of volume fraction $\phi$ from both simulation and experiment. Simulation results include both previously measured values from runs at $P e=0.01$ (Phung 1993) and values determined in this work from an equilibrium Green-Kubo analysis.

remains roughly constant and equal to the high-frequency dynamic viscosity, $\eta_{\infty}^{\prime}$, throughout the shear thinning process and then increases for $P e>10$. Thus, the total viscosity goes through two regions of behaviour, a shear-thinning region at low $P e$ and a shear-thickening region at high $P e$. The constancy of the hydrodynamic viscosity and the shear thinning of the Brownian viscosity has been observed in the stress jump experiments of Mackay \& Kaffashi (1995) and the optical measurements of Bender \& Wagner (1996). The uncertainty in the viscosities is shown in table 2. 




FIGURE 2. Peclet number dependence of the different contributions to the relative viscosity of hard-sphere suspensions at $\phi=0.45$ and $N=27$ determined by Stokesian Dynamics. The horizontal lines on the far left represent the $P e \rightarrow 0$ limits independently determined by an equilibrium Green-Kubo analysis.

Shear thinning can be explained in the following manner: the Brownian stress arises from the flow-induced deformation of the equilibrium structure - particles diffuse against the flow towards their unstressed configuration and the resultant stress is directly proportional to the deformation. This deformation is known to be linear in the Péclet number for very small Péclet numbers. Since the viscosity of a suspension is simply the stress non-dimensionalized by $\eta \dot{\gamma}$, the viscosity scales as the 'deformation' $/ P e$, hence $\eta_{r}^{B} \sim O(1)$ as $P e \rightarrow 0$. In order for the Brownian viscosity to remain constant as $P e$ is increased, the flow-induced deformation must continue to increase linearly with $P e$. However, the Péclet number is also the ratio of the relaxation time due to diffusion $a^{2} / D$ to the time scale of the flow $1 / \dot{\gamma}$, and as the Péclet number increases the particle motion cannot keep up with the flow and the structural deformation saturates. Hence the Brownian viscosity decreases as $P e \rightarrow \infty$. Recent theoretical work (Brady \& Morris 1997) predicts the Brownian viscosity to decay as $1 / P e$, which is in fair agreement with the results of the simulations (cf. table 2).

The hydrodynamic stress arises because the particles are rigid and do not deform as fluid elements. For the volume fractions studied here, $0.316 \leqslant \phi \leqslant 0.49$, the hydrodynamic viscosity varies little between a random and a regular array and is roughly the same for any 'well-dispersed' structure. Although the structure evolves quite significantly at low Péclet numbers (cf. figure 20), the suspension remains 'well-dispersed' and the hydrodynamic viscosity is constant. In simple shear flow particles are pushed together along the compressive axes of the flow, while the action of Brownian motion is to keep particles apart and well dispersed. (In fact it is the $\boldsymbol{\nabla} \cdot \boldsymbol{R}_{F U}^{-1}$ term in (4)-(5) that acts as a repulsive radial force and balances the hydrodynamic squeezing force 


\begin{tabular}{cccccccr}
\hline Pe & $N$ & $\Delta t$ & $\begin{array}{c}\text { NSTEPS } \\
\left(\times 10^{3}\right)\end{array}$ & $\eta_{r}^{H}$ & $\eta_{r}^{B}$ & $N_{1} / \eta \dot{\gamma}$ & $N_{2} / \eta \dot{\gamma}$ \\
& & & & & \\
$0.01^{*}$ & 27 & $5 \times 10^{-4}$ & 1000 & $4.61 \pm 0.01$ & $9.0 \pm 9.1$ & $20.7 \pm 14.9$ & $-10.43 \pm 5.10$ \\
0.10 & 27 & $5 \times 10^{-4}$ & 1000 & $4.54 \pm 0.05$ & $7.00 \pm 1.11$ & $4.13 \pm 3.08$ & $-3.07 \pm 2.94$ \\
0.30 & 27 & $5 \times 10^{-4}$ & 200 & $4.60 \pm 0.16$ & $5.79 \pm 0.69$ & $5.01 \pm 2.72$ & $-3.41 \pm 2.59$ \\
1.00 & 27 & $5 \times 10^{-4}$ & 200 & $4.78 \pm 0.13$ & $3.70 \pm 0.27$ & $0.95 \pm 0.57$ & $-2.12 \pm 1.11$ \\
3.00 & 27 & $5 \times 10^{-4}$ & 200 & $4.91 \pm 0.29$ & $2.00 \pm 0.27$ & $-0.45 \pm 0.17$ & $-1.21 \pm 0.77$ \\
5.00 & 27 & $5 \times 10^{-4}$ & 200 & $5.07 \pm 0.27$ & $1.46 \pm 0.21$ & $-0.65 \pm 0.57$ & $-1.13 \pm 0.33$ \\
7.00 & 27 & $5 \times 10^{-4}$ & 200 & $5.28 \pm 0.18$ & $1.23 \pm 0.07$ & $-0.53 \pm 0.12$ & $-1.32 \pm 0.14$ \\
10.00 & 27 & $5 \times 10^{-4}$ & 200 & $5.59 \pm 0.47$ & $1.07 \pm 0.21$ & $-0.25 \pm 0.76$ & $-1.39 \pm 0.47$ \\
20.00 & 27 & $5 \times 10^{-4}$ & 200 & $6.30 \pm 0.30$ & $0.76 \pm 0.09$ & $-1.20 \pm 0.67$ & $-1.43 \pm 0.34$ \\
$10^{2}$ & 27 & $5 \times 10^{-4}$ & 200 & $7.56 \pm 0.41$ & $0.22 \pm 0.03$ & $-1.37 \pm 0.38$ & $-1.62 \pm 0.56$ \\
$2 \times 10^{2}$ & 27 & $5 \times 10^{-4}$ & 200 & $8.02 \pm 0.28$ & $0.12 \pm 0.01$ & $-1.09 \pm 0.41$ & $-1.87 \pm 0.36$ \\
$10^{3}$ & 27 & $5 \times 10^{-4}$ & 200 & $9.25 \pm 0.59$ & $0.029 \pm 0.004$ & $-1.81 \pm 0.79$ & $-1.61 \pm 0.38$ \\
$10^{4}$ & 27 & $2.5 \times 10^{-4}$ & 400 & $11.64 \pm 1.11$ & $0.004 \pm 0.001$ & $-1.12 \pm 1.27$ & $-2.44 \pm 0.51$ \\
& & & & & & \\
$0.01^{*}$ & 63 & $5 \times 10^{-4}$ & 80 & $4.42 \pm 0.01$ & $10.2 \pm 8.2$ & & \\
$0.43^{*}$ & 63 & $10^{-3}$ & 40 & $4.78 \pm 0.02$ & $6.48 \pm 0.13$ & $0.86 \pm 0.10$ & $-1.34 \pm 0.11$ \\
10.0 & 63 & $5 \times 10^{-4}$ & 200 & $5.77 \pm 0.21$ & $1.10 \pm 0.10$ & $-0.42 \pm 0.26$ & $-1.30 \pm 0.31$ \\
$10^{3}$ & 63 & $5 \times 10^{-4}$ & 200 & $8.87 \pm 0.18$ & $0.026 \pm 0.001$ & $-1.66 \pm 0.42$ & $-1.63 \pm 0.27$ \\
$0.43^{*}$ & 123 & $5 \times 10^{-4}$ & 80 & 4.65 & 5.454 & 1.043 & -1.313 \\
10.0 & 123 & $10^{-3}$ & 60 & 5.696 & 1.094 & -0.340 & -1.146 \\
$10^{3}$ & 123 & $10^{-3}$ & 50 & 8.954 & 0.028 & -0.340 & -1.683
\end{tabular}

TABle 2. Simulations data with $\phi=0.45$. Column 1 is the Péclet number and column 2 is the number of particles. Columns 3 and 4 are the time step and the total number of time steps. Columns 5 and 6 give the Brownian and hydrodynamic contributions to the shear viscosity (normalized by the solvent viscosity). And columns 7 and 8 give the first and second normal stress differences. Initial particle configurations of all the runs are random. The error estimates were determined by dividing a simulation run up into statistically independent subintervals (5-10) and comparing the averages for each interval (see Phung 1993). "Data obtained from Phung (1993).

along the compressive axes (Bossis \& Brady 1989).) At high Péclet numbers hydrodynamic forces dominate everywhere except in a thin $O\left(P e^{-1}\right)$ boundary layer adjacent to particle surfaces where there is a balance of hydrodynamic and Brownian forces (Brady \& Morris 1997). Once the Péclet number exceeds $O(10)$ hydrodynamic forces are capable of pushing two particles close enough together for the strong lubrication forces to come into play; lubrication forces are singular near contact as $1 /(r-2 a)$, with this singularity being felt when $r-2 a \leqslant 10^{-2} a$. As the Péclet number is increased particles are progressively 'stuck' together by the strong lubrication forces and form non-compact aggregates or clusters. As shown in earlier work on monolayers (Bossis \& Brady 1989) the cluster size grows as the Péclet number increases. Associated with a growing cluster size is an increase in the contact value of the pair-distribution function reported in table 3 . The increased contact value is also evident in the sharpening of the first nearest neighbour peak in figure 20.

Although the stress is purely hydrodynamic at large Péclet number, this does not mean that Brownian motion plays no role. The limit $P e \rightarrow \infty$ is singular and the residual effect of Brownian motion at particle contact limits the ultimate size of the clusters. The relative tangential motion of two particles is resisted by a weak logarithmic dependence on particle separation and the small amount of Brownian motion provides a means for two near touching particles to move relative to one another and break the connectivity of the cluster, dramatically affecting the viscosity 


\begin{tabular}{ccccccc}
\hline$P e$ & $N$ & $\langle g(2)\rangle_{\Omega}$ & $D_{0}^{s}$ & $D_{0}^{r}$ & $D_{y y}$ & $D_{z z}$ \\
$0.00^{*}$ & 27 & & 0.210 & 0.604 & 0.059 & 0.055 \\
$0.01^{*}$ & 27 & 4.20 & 0.172 & 0.553 & 0.068 & 0.058 \\
0.10 & 27 & 4.47 & 0.177 & 0.554 & 0.069 & 0.096 \\
0.30 & 27 & 4.82 & 0.174 & 0.549 & 0.115 & 0.083 \\
1.00 & 27 & 5.80 & 0.168 & 0.534 & 0.146 & 0.151 \\
3.00 & 27 & 7.73 & 0.159 & 0.514 & 0.311 & 0.257 \\
5.00 & 27 & 9.22 & 0.154 & 0.503 & 0.636 & 0.425 \\
7.00 & 27 & 10.9 & 0.149 & 0.492 & 0.846 & 0.405 \\
10.00 & 27 & 13.6 & 0.140 & 0.473 & 0.688 & 0.452 \\
20.00 & 27 & 20.8 & 0.129 & 0.446 & 1.247 & 5.457 \\
$10^{2}$ & 27 & 39.0 & 0.105 & 0.383 & 2.349 & 3.374 \\
$2 \times 10^{2}$ & 27 & 43.1 & 0.099 & 0.364 & 6.788 & 23.58 \\
$10^{3}$ & 27 & 49.9 & 0.088 & 0.321 & 47.27 & \\
$10^{4}$ & 27 & 59.2 & 0.069 & 0.256 & 644.5 & \\
$0.01^{*}$ & 63 & 4.51 & 0.204 & 0.558 & & \\
$0.43^{*}$ & 63 & 4.46 & 0.184 & 0.541 & & \\
$0.43^{*}$ & 123 & 4.94 & 0.204 & 0.551 & & \\
10.00 & 123 & 14.1 & 0.163 & 0.471 & & \\
$10^{3}$ & 123 & 50.3 & 0.101 & 0.317 & &
\end{tabular}

TABLE 3. Same runs as in table 1 but showing the angularly-averaged pair-distribution function at contact and the self-diffusivities normalized by the isolated particle Brownian diffusivities. The error on the short-time self-diffusivities is \pm 1 in the last digit, and for the long-time self-diffusivities, the error is about $30 \%$. "Data obtained from Phung (1993).

(Bossis, Meunier \& Brady 1991). Previously reported results (Phung et al. 1996) in the pure hydrodynamic limit $\left(P e^{-1} \equiv 0\right)$ have not been affected by the nondimensionalization error as they include no Brownian motion. These simulations in the pure hydrodynamic limit failed to reach a steady state. A typical run in this regime would proceed in time with the viscosity slowly increasing until suddenly a large cellspanning cluster would form jamming particles together, sending the viscosity to enormous values, and halting the integration. Reducing the time step allowed only a very small additional advance in time. Increasing the size of the unit cell delayed the onset of the catastrophic shear thickening but did not eliminate it. This behaviour happens at moderate to large concentrations and it is not known if there is a volume fraction below which a spanning cluster no longer forms.

The problems with $\left(\mathrm{Pe}^{-1} \equiv 0\right)$ simulations have also been discussed by Ball \& Melrose (1995), Melrose \& Ball (1995), and Dratler \& Schowalter (1996). In fact, a high-Péclet-number asymptote for the viscosity was not obtained for any of the volume fractions studied here. The size of the time step, $\Delta t$, for the largest Péclet number $\left(10^{4}\right)$ runs for each volume fraction are $2.5 \times 10^{-4}$ compared to $5 \times 10^{-4}$ for most of the other runs. This is because $P e=10^{4}$ runs with $\Delta t=5 \times 10^{-4}$ displayed the same problems with growing clusters, diverging viscosities and halted integration as the aforementioned $\left(P e^{-1} \equiv 0\right)$ runs. The most likely explanation for this is that the larger time step is unable to properly resolve the $P e^{-1}$ boundary layer as the Péclet number gets large. One can see that as $P e \rightarrow \infty$, the time step necessary to capture the proper physics of the boundary layer would have to scale as $P e^{-1}$, which becomes an unreasonable constraint numerically and is consistent with the pathological behaviour of simulations run in the pure hydrodynamic limit $\left(P e^{-1} \equiv 0\right)$. Previous simulations 


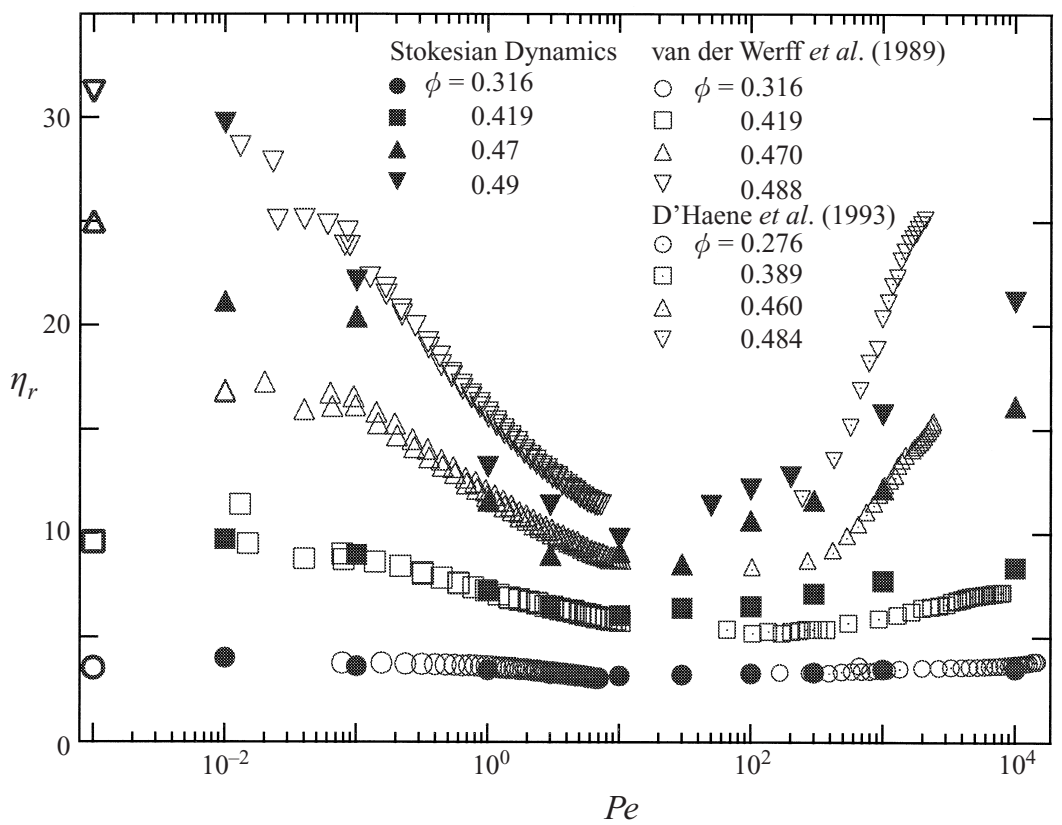

FIGURE 3. Comparison of the relative viscosity of hard-sphere suspensions determined by Stokesian Dynamics as a function of the Péclet number $P e$ with the experimental results (open symbols) of van der Werff \& de Kruif (1989) at low $P e$ and D'Haene et al. (1993) at high $P e$. The thick open symbols on the far left represent the $P e \rightarrow 0$ limits independently determined by an equilibrium Green-Kubo analysis.

with the erroneous high-Pe evolution equation were able to run at Péclet numbers as high as $10^{6}$ (Phung et al. 1996; Phung 1993). The random steps in those simulations were too small compared to the deterministic $\nabla \cdot \boldsymbol{R}_{F U}^{-1}$ term. The relative 'enhancement' of the $\nabla \cdot \boldsymbol{R}_{F U}^{-1}$ term in those simulations caused the particles to act as if there was an additional radially repulsive force between them. The ability of those runs to achieve a steady state at higher Péclet numbers is consistent with the increased robustness of $P e^{-1} \equiv 0$ simulations with repulsive interparticle forces (Brady \& Bossis 1985; Dratler \& Schowalter 1996; Yurkovetsky 1998).

This sensitivity to small-scale surface interactions and the singular nature of the $P e \rightarrow \infty$ limit have important implications for the interpretation of experimental viscosity measurements at high concentrations and shear rates because seemingly small factors can dramatically influence the results. It may also explain why measurements of viscosity at high concentrations in the pure hydrodynamic limit show a large amount of scatter (an order of magnitude) from one researcher to the next, although the reproducibility for an individual researcher was good (Thomas 1965). It also suggests that if short-range surface effects can be controlled and the Péclet number is made large enough the clusters should grow to the size of the experimental apparatus and the behaviour should depend on the size of the measuring cell.

In figure 3 we compare the simulation viscosities with the experiments of van der Werff \& de Kruif (1989) on monodisperse spherical silica particles that have been shown to behave to a very good approximation as hard spheres. The simulation and experimental viscosities are in good quantitative agreement considering the strong dependence of the viscosity on volume fraction at high volume fraction and the uncertainty in precisely determining the experimental volume fraction. The 


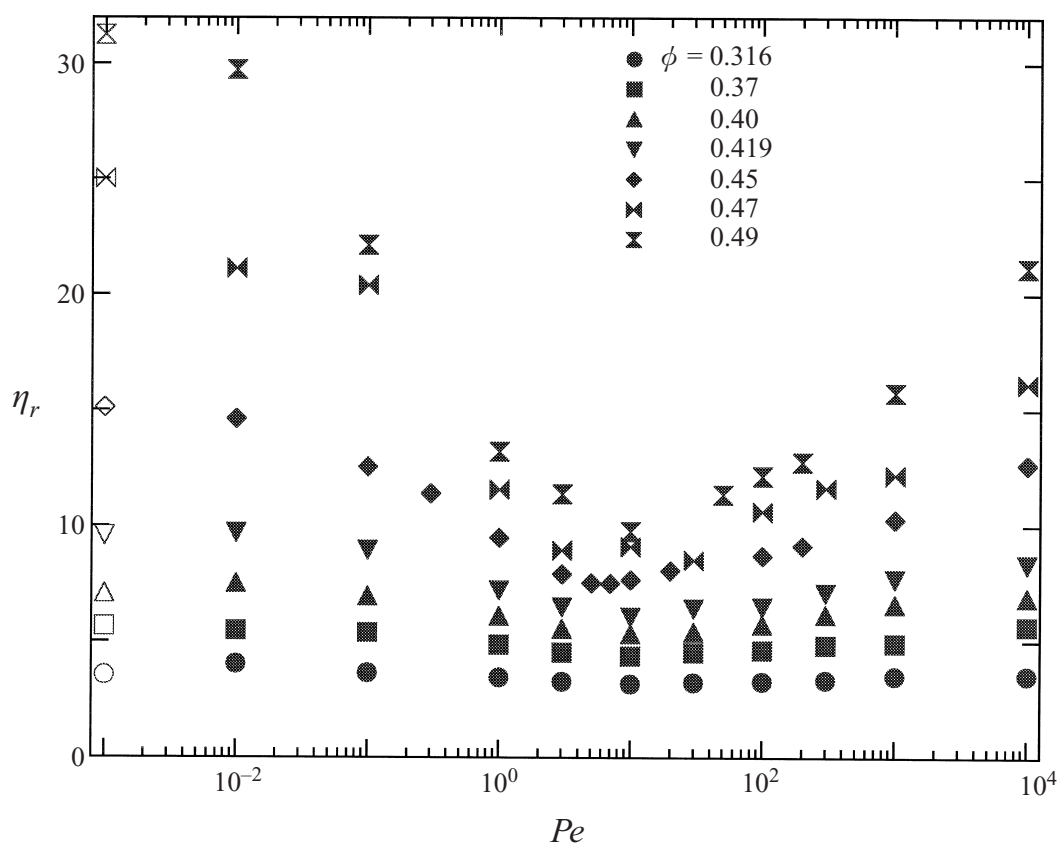

FIGURE 4. The relative viscosity, $\eta_{r}=1+\eta_{r}^{B}+\eta_{r}^{H}$, as a function of Péclet number $P e$ for the range of volume fraction $0.316 \leqslant \phi \leqslant 0.49$ with $N=27$. The open symbols on the far left represent the $P e \rightarrow 0$ limits independently determined by an equilibrium Green-Kubo analysis.

experiments do not show shear thickening as their Péclet numbers were too low. Additional experiments by the same group up to $P e=1200$ did not display shear thickening. It may be that shear thickening occurs at a slightly higher Péclet number in these systems, possibly due to the fact that the silica particles are coated with short hydrocarbon chains and therefore are weakly repulsive when brought into near contact. However, shear thickening due solely to the growth of clusters as first predicted in the monolayer simulations has been observed experimentally by D'Haene et al. (1993) and Bender \& Wagner $(1995,1996)$. As seen in figure 3 the PMMA suspensions of D'Haene et al. (1993) show shear thickening at high Péclet number and are in reasonable agreement with the simulation results. The experimental results at the highest volume fraction thicken more rapidly than the simulations, which may be due to the collapse of the stabilizing polymer chains that have been grafted onto the particle surfaces. Or it may simply reflect the extreme volume fraction sensitivity of the phenomena and the difficulty in accurately measuring the volume fraction.

Figure 4 shows the total relative viscosity plotted as a function of Péclet number for all volume fractions studied. More detailed figures showing the separate hydrodynamic and Brownian contributions for each volume fraction can be found in Foss (1999); the trends are identical to those shown in figure 2 for $\phi=0.45$. The uncertainty in the viscosities is comparable to that shown in table 2 for $\phi=0.45$. To examine the shear thinning and thickening behaviour and to compare with existing theories, it is important to separate out the contribution to the viscosity resulting from the hydrodynamic interactions that are present in the equilibrium configuration - the high-frequency dynamic viscosity $\eta_{\infty}^{\prime} / \eta=1+\eta_{r}^{H}(P e=0)$. The remaining viscosity, $\Delta \eta / \eta=\eta_{r}-\eta_{\infty}^{\prime} / \eta$, arises from particle interactions (hydrodynamic and Brownian) in the non-equilibrium structure induced by the flow. Theories have been advanced to predict $\Delta \eta$ as a function of concentration and shear rate which we now discuss. 
Let us first examine the shear-thickening regime $P e \geqslant O(10)$. Brady \& Morris (1997) showed that the limit of large Péclet number is singular with an $O\left(P e^{-1}\right)$ thin boundary layer at particle-particle contact in which Brownian and hydrodynamic forces balance. Brady \& Morris also showed that the hydrodynamic contribution to the stress from this boundary layer, which is the dominant contribution at high $P e$, scales as $S_{b . l}^{H} \sim \dot{\gamma} \eta_{\infty}^{\prime}(\phi) \phi^{2} g^{\infty}(2 ; \phi)$, where $g^{\infty}(2 ; \phi)$ is the pair-distribution function just outside the boundary layer at the high Péclet number $(\infty)$ and the concentration of interest, and $\eta_{\infty}^{\prime}(\phi)$ is the (dimensional) high-frequency dynamic viscosity. A simple physical explanation for this scaling is the following.

The stress from the boundary layer is a two-body effect and near contact the hydrodynamic stress can be estimated from the moment of the hydrodynamic shear force:

$$
\boldsymbol{S}_{b . l .}^{H} \sim-n^{2} \int_{b . l .} \boldsymbol{r} \boldsymbol{F}^{\text {shear }} g(\boldsymbol{r}) \mathrm{d} \boldsymbol{r} .
$$

The hydrodynamic shear force scales as $\boldsymbol{F}^{\text {shear }} \sim-3 \pi \eta_{\infty}^{\prime}(\phi) a^{2} \dot{\gamma} \times \hat{\boldsymbol{r}}(\hat{\boldsymbol{r}} \cdot \boldsymbol{E} \cdot \hat{\boldsymbol{r}})$, where $\hat{\boldsymbol{r}}$ is the unit vector along the line of centres of the two particles, and the angle brackets on $\boldsymbol{E}$ denoting a suspension average have been dropped. In a concentrated suspension the shear force is enhanced over that for two particles alone in the fluid in essence because it acts through the suspension, while the resistance to this squeezing force is through the solvent as only solvent can be in the gap between two near touching particles. Stokesian Dynamics simulations at high Pe (Brady \& Bossis 1985; Bossis, Brady \& Mathis 1988) bear this out and show that the relative velocity of two particles near contact is enhanced in a concentrated suspension and an estimate for the $\phi$-dependence of that enhancement is $\eta_{\infty}^{\prime}(\phi)$.

Although the boundary-layer thickness is small, $O\left(P e^{-1}\right)$, and therefore one might expect that the contribution in (13) would be small, along the compressive axes of the flow, $\hat{\boldsymbol{r}} \cdot \boldsymbol{E} \cdot \hat{\boldsymbol{r}}<0$ (cf. figure 20), the pair-distribution function within the boundary layer is large, $O(P e)$. Brady \& Morris (1997) show that along the compressive axes $g_{b . l .}(\boldsymbol{r}) \sim P \operatorname{eg}^{\infty}(2 ; \phi) \bar{g}(\theta, \varphi)$, where $g^{\infty}(2 ; \phi)$ is the value of the pair-distribution function just outside the boundary layer, and $\bar{g}(\theta, \varphi)$ is the $O(1)$ angular variation within the boundary layer. Thus, the integral (13) for the stress from the boundary layer is

$$
\boldsymbol{S}_{b . l .}^{H} \sim \eta_{\infty}^{\prime}(\phi) \dot{\gamma} \phi^{2} g^{\infty}(2 ; \phi) \int_{\hat{\boldsymbol{r}} \cdot \mathbf{E} \cdot \hat{\boldsymbol{r}}<0} \hat{\boldsymbol{r}} \hat{\boldsymbol{r}}(\hat{\boldsymbol{r}} \cdot \boldsymbol{E} \cdot \hat{\boldsymbol{r}}) \bar{g}(\theta, \varphi) \mathrm{d} \Omega,
$$

where $\mathrm{d} \Omega$ represents the solid angle and the limits of angular integration are restricted to regimes where $g(\boldsymbol{r}) \sim O(P e)$, i.e. the compressive axes. For a simple radial-balance approximation, Brady \& Morris show that $\bar{g}(\theta, \varphi) \propto-\hat{\boldsymbol{r}} \cdot \boldsymbol{E} \cdot \hat{\boldsymbol{r}}$. The expression for the boundary-layer stress (14) is only approximate due to the approximations that have been made for the shear forces, etc. Further, there are other contributions to the hydrodynamic stress from particle interactions outside the boundary layer and along the extensional axes, but we expect these to be no larger and therefore (14) to give a reasonable estimate of the hydrodynamic stress over and above the high-frequency dynamic viscosity (which is associated with the disordered structure outside the boundary layer).

In addition to an estimate of the hydrodynamic stress, the analysis of Brady \& Morris (1997) also shows that the Péclet number needs to be rescaled in the shearthickening regime. Since the Péclet number is the ratio of shear forces to Brownian forces, the rescaled Péclet number is simply $\overline{P e}=6 \pi \eta_{\infty}^{\prime}(\phi) a^{3} \dot{\gamma} / k T=P e \eta_{\infty}^{\prime}(\phi) / \eta$. From figure 4 one sees that the higher the concentration the earlier shear thickening begins, in agreement with this rescaling. 


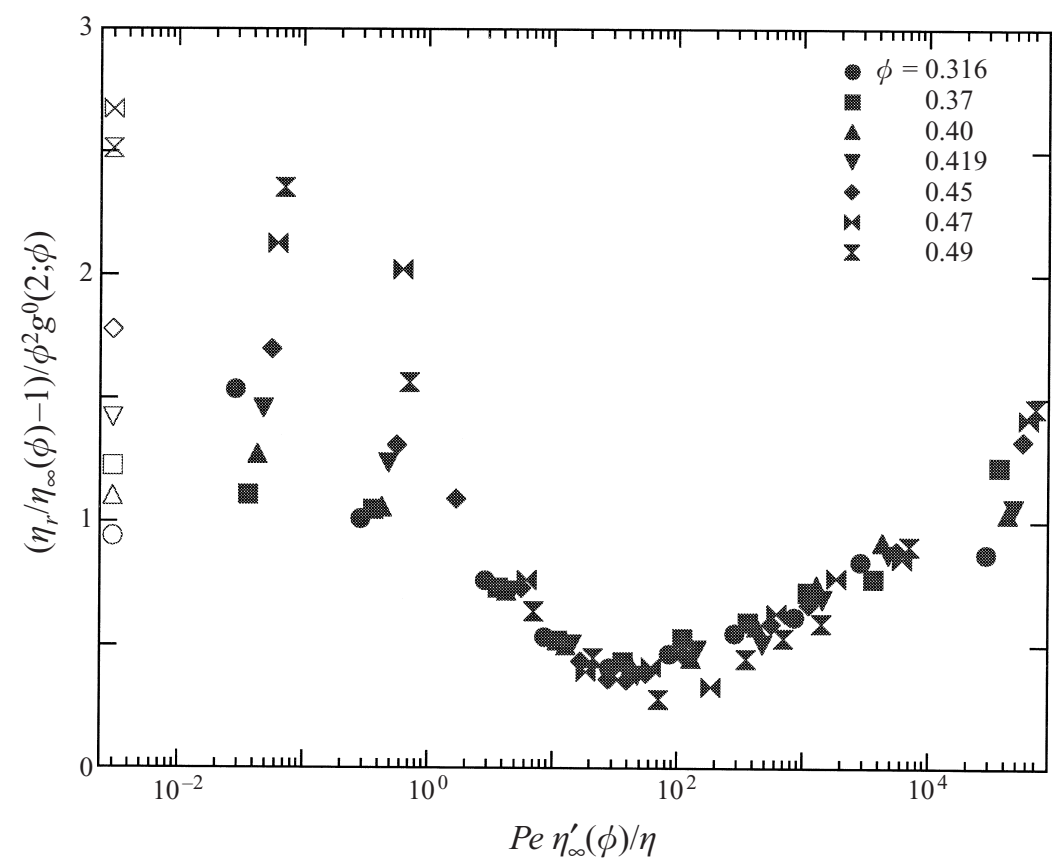

FIGURE 5 . The difference of the total viscosity, $\eta_{r}$, from its equilibrium hydrodynamic contribution, $\eta_{\infty}^{\prime}$, scaled by $\eta_{\infty}^{\prime} \phi^{2} g^{0}(2)$ is plotted as a function of rescaled Péclet number $\overline{P e}=P e \eta_{\infty}^{\prime} / \eta$ for volume fractions $0.316 \leqslant \phi \leqslant 0.49$ with $N=27$. The open symbols on the far left represent the $P e \rightarrow 0$ limits independently determined by an equilibrium Green-Kubo analysis.

The last item needed is the contact value of the pair-distribution function outside the boundary layer $g^{\infty}(2 ; \phi)$. On this the theory of Brady \& Morris (1997) is silent. We could use simulation results, but then that would not result in a predictive theory. Instead, we have chosen to use the equilibrium pair distribution for hard spheres, $g^{0}(2 ; \phi)$, which is a known function of $\phi$, for example from the Carnahan-Starling equation of state:

$$
g^{0}(2 ; \phi)=\frac{1-\frac{1}{2} \phi}{(1-\phi)^{3}}, \quad \phi<0.50 .
$$

This is, of course, not correct, but it should give a reasonable estimate over the range of $\phi$ investigated here. Near close packing, this would not necessarily be a good estimate as $g^{0}(2)$ diverges at random close packing $\phi_{r c} \approx 0.63$ (not with the Carnahan-Starling equation, however), while $g^{\infty}(2)$ may diverge in a different manner and at different maximum concentration.

These arguments suggest that a plot of

$$
\frac{\Delta \eta}{\eta_{\infty}^{\prime}(\phi)} \frac{1}{\phi^{2} g^{0}(2 ; \phi)}=\frac{\eta(\phi ; \overline{P e})-\eta_{\infty}^{\prime}(\phi)}{\eta_{\infty}^{\prime}(\phi) \phi^{2} g^{0}(2 ; \phi)}
$$

versus $\overline{P e}=P e \eta_{\infty}^{\prime}(\phi) / \eta$ should collapse the shear-thickening behaviour to a single universal curve for all $\phi$. Figure 5 shows the data for all shear rates in figure 4 replotted according to this scaling estimate. The shear-thickening data collapse quite well, showing that $\overline{P e}$ is the appropriate scale for the shear rate and that the boundary layer scaling with the equilibrium pair-distribution function $g^{0}(2 ; \phi)$ collapse the magnitude well. 


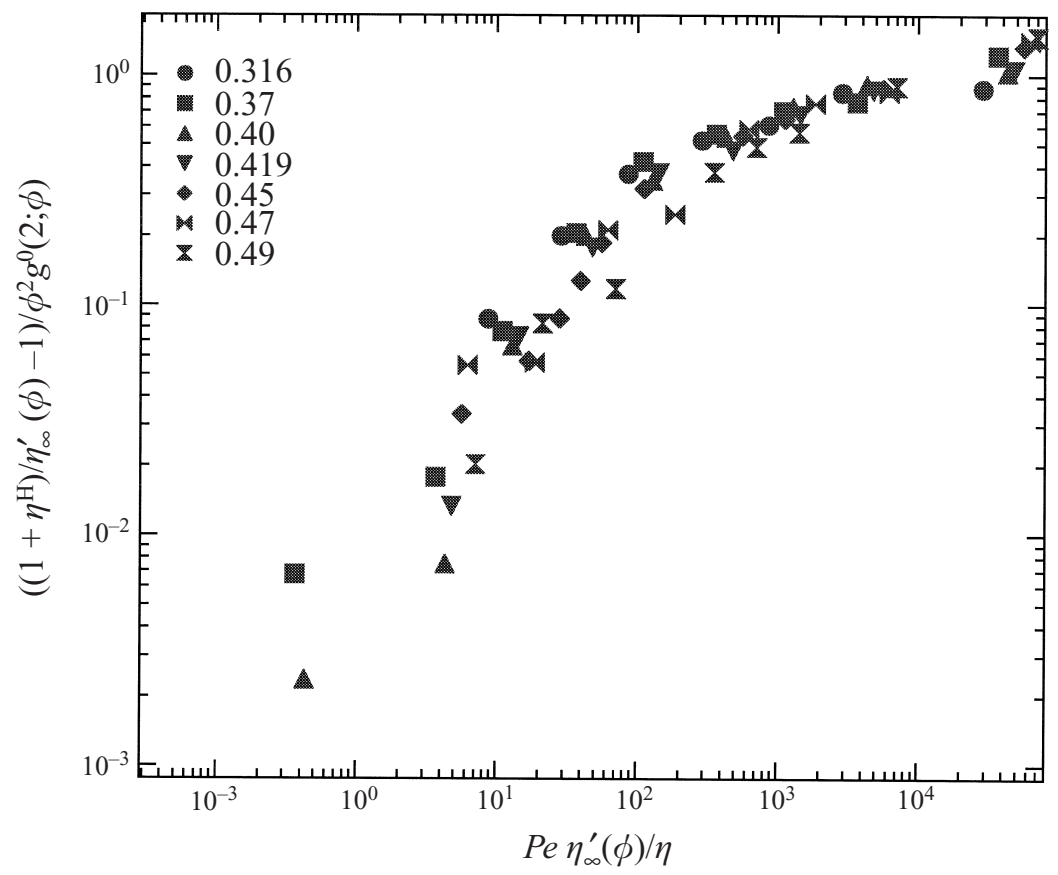

FIGURE 6 . The difference of the total hydrodynamic contribution to the viscosity, $1+\eta^{H}$, from its equilibrium contribution, $\eta_{\infty}^{\prime}$, scaled by $\eta_{\infty}^{\prime} \phi^{2} g^{0}(2) / \eta$ is plotted as a function of rescaled Péclet number $\overline{P e}=P e \eta_{\infty}^{\prime} / \eta$ for volume fractions $0.316 \leqslant \phi \leqslant 0.49$ with $N=27$.

Figure 5 also shows that the viscosity does not approach an asymptote as $P e \rightarrow \infty$. This is shown more clearly in figure 6 where only the scaled hydrodynamic viscosity (over and above the high-frequency dynamic viscosity) is plotted versus the scaled Péclet number. This behaviour confirms the results of Ball \& Melrose (1995) and Dratler \& Schowalter (1996) that in the pure hydrodynamic limit $(P e \rightarrow \infty$ for hard spheres) a steady shear viscosity does not exist because hydrodynamic clusters form whose size diverges as $P e \rightarrow \infty$. The growth with $\overline{P e}$ in figure 6 is very weak, approximately as $(\ln \overline{P e})$, although caution must be exercised in drawing firm conclusions because the system sizes are small and the periodicity would likely affect the detailed behaviour. One would expect, for example, that there would be a critical volume fraction below which infinite clusters would not form and an asymptotic viscosity would exist.

In figure 5 we plotted the data for all shear rates according to the high-shearrate scaling behaviour and, while the spread in the shear-thinning data is reduced, this scaling does not appear to completely collapse the shear-thinning data. At low shear rates it is the Brownian contribution to the stress that is responsible for shearthinning. To obtain an estimate for the shear-thinning behaviour, we start by rewriting the Brownian contribution to the stress in the equivalent form

$$
n\left\langle\boldsymbol{S}^{B}\right\rangle=-n^{2} k T a \int_{r=2 a} \hat{\boldsymbol{r}} \hat{r} g(\boldsymbol{r}) \mathrm{d} S+n k T\left\langle\boldsymbol{R}_{S U} \cdot \boldsymbol{R}_{F U}^{-1} \cdot \nabla \ln P_{N}\right\rangle,
$$

which can be obtained from $(10 c)$ by introducing the probability density for the $\mathrm{N}$ particle configuration $P_{N}(\boldsymbol{x}, t)$ and integrating by parts (Brady 1993a). This form for $\left\langle\boldsymbol{S}^{B}\right\rangle$ is particularly convenient in that it separates out the contribution for particles in 
contact (the first integral), which is of exactly the same form as in atomic or molecular hard spheres, from the remainder which is due to hydrodynamic interactions $\left(\boldsymbol{R}_{S U}\right)$ among particles.

Here, we focus on the contact integral which has been shown to give a good estimate of the Brownian stress at high densities (Brady 1993b). The low-shear viscosity can be extracted from this integral by using the first perturbation due to flow to the equilibrium structure, $f(\boldsymbol{r})$, defined by $g(\boldsymbol{r})=g^{0}(\boldsymbol{r})[1+f(\boldsymbol{r})]$. The equilibrium stress is just the isotropic osmotic pressure, $-\Pi^{0} I$. The first contribution to the viscosity is due to the $O(P e)$ correction to the microstructure and results in a constant low-Pe Newtonian plateau with viscosity equal to the zero-shear viscosity, $\eta_{0}$; thus we can write

$$
f(\boldsymbol{r})=\widehat{P e} \hat{f}(\boldsymbol{r}),
$$

where $\hat{f}(\boldsymbol{r})$ is independent of the flow strength and $\widehat{P e}=P e / \hat{D}(\phi)$, where $\hat{D}(\phi)$ is the characteristic diffusivity non-dimensionalized by the Stokes-Einstein diffusivity, $D$. Substituting the expression for $f(\boldsymbol{r})$ into the contact integral in (15) gives the following expression for the deviatoric part of the Brownian stress:

$$
n\left\langle\boldsymbol{S}^{B}\right\rangle+\Pi^{0} \boldsymbol{I}=-\frac{27}{2 \pi} \eta \dot{\gamma} \phi^{2} \frac{g^{0}(2 ; \phi)}{\hat{D}(\phi)} \int \hat{\boldsymbol{r}} \hat{\boldsymbol{r}} \hat{f}(2 ; \theta, \varphi) \mathrm{d} \Omega .
$$

The characteristic diffusivity is determined by the appropriate relaxation time for rheological response: $a^{2} / \hat{D}(\phi) D$. With this scaled Péclet number, all of the $P e$-dependence of the microstructure at all volume fractions is included in $\widehat{P e}$. In the dilute limit, all particle diffusivities are equal to the diffusivity of an isolated particle, $D$. Thus, $\hat{D}=1$ and (16) reduces to the the Brownian stress as previously calculated by various twoparticle theories (Batchelor 1977; Brady \& Vicic 1995; Lionberger 1998; Vicic 1999).

The precise nature of $\hat{D}(\phi)$ is still an open question. Many quantities have been suggested. Brady (1993b) suggests using the equilibrium short-time self-diffusivity, $\hat{D}(\phi)=D_{0}^{s}(\phi) / D \approx \eta / \eta_{\infty}^{\prime}(\phi)$, as this incorporates the primary effect of the hydrodynamic interactions. The shear-thinning behaviour from this scaling is the same as the one used above to collapse the shear-thickening data in figure 5. As noted before, this scaling is effective in reducing the spread in the data, but a sufficient collapse is not obtained. One can see this more clearly when the same scaling is used for only the Brownian contribution to the stress in figure 7. Nevertheless, use of the short-time self-diffusivity is successful in removing all of the hydrodynamics, and thus data from suspensions with and without hydrodynamic interactions should be indistinguishable with this scaling (Foss \& Brady 2000).

Apparently, simply scaling out the hydrodynamics is not sufficient, and there appears to be another contribution to $\hat{D}(\phi)$ from the relaxation of the dynamic microstructure. A simple choice would be to use the equilibrium long-time selfdiffusivity, $\hat{D}(\phi)=D_{\infty}^{s}(\phi) / D$. A collapse of the viscosity data using this scaling is shown in figure 8 . We have used the values of $\hat{D}_{\infty}^{s}(\phi)$ obtained from these simulations (cf. §3.2). This choice of $\hat{D}(\phi)$ arguably works better than that used in figure 5, especially at the higher volume fractions. (Note the scale of the ordinate in figures 5 and 8.) Note that the collapse of the shear-thickening data is much worse than before, as the arguments presented here concern only the Brownian stress. A plot of only the Brownian contribution to the viscosity is shown in figure 9.

Another diffusivity often suggested as a good candidate for $\hat{D}(\phi)$ is the wavelengthdependent collective diffusivity evaluated at the peak of the structure factor, $D^{c}\left(k_{\max }\right) / D$ (Verberg, de Schepper \& Cohen 1997; Pusey et al 1997), as this rep- 


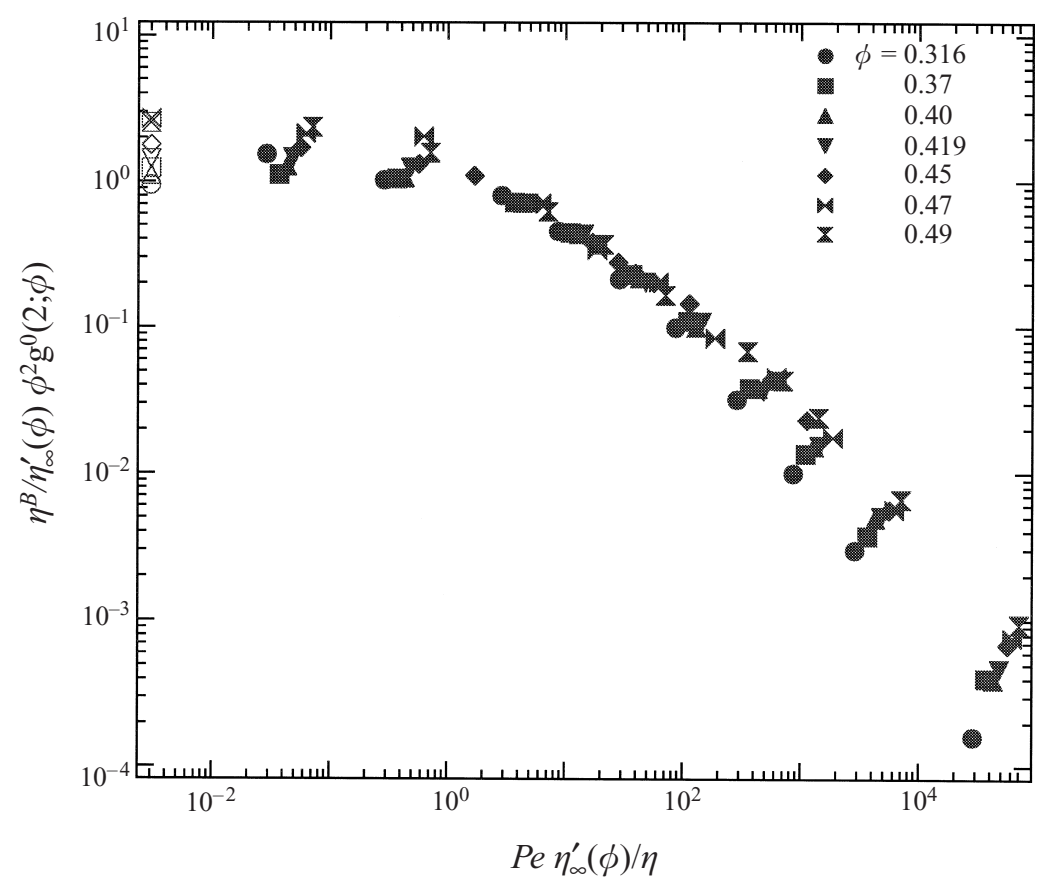

FIGURE 7. The Brownian contribution to the viscosity, $\eta^{B}$, scaled by $\eta_{\infty}^{\prime} \phi^{2} g^{0}(2) / \eta$ and plotted as a function of rescaled Péclet number $\overline{P e}=P e \eta_{\infty}^{\prime} / \eta$ for volume fractions $0.316 \leqslant \phi \leqslant 0.49$ with $N=27$. The open symbols on the far left represent the $P e \rightarrow 0$ limits independently determined by an equilibrium Green-Kubo analysis.

resents relaxation of the dominant 'cage' structure and is also the slowest rate of structural decay present. No plots of this possible collapse are shown as we have not calculated $D^{c}\left(k_{\max }\right) / D$ in our simulation.

There may be no simple relationship between $\hat{D}(\phi)$ and a previously known diffusivity. The aforementioned relationship between $\hat{D}(\phi)$ and $D_{0}^{s}(\phi) / D$ was first suggested by the experimental data of van der Werff et al. (1989). Although, in general, this collapse of our data is unsuccessful, it does appear valid for the lower volume fractions $(\phi \leqslant 0.40)$. Data from experiments by Shikata \& Pearson (1994) show that the relationship between $\hat{D}(\phi)$ and $D_{0}^{s}(\phi) / D_{0}$ holds up to $\phi=0.50$ before it breaks down. They suggest that at higher volume fractions, other relaxation processes, perhaps associated with a glass transition, become increasingly important at high densities. Pusey et al. (1997) also note a particularly strong slowing down of the structural relaxation for $\phi>0.40$. Very recent work on mode-coupling theory (Banchio, Bergenholtz \& Nägele 1999) makes a strong case for glass-like behaviour at high concentrations.

\subsubsection{Normal stress differences}

The first and second normal stress differences are defined by

$$
\begin{aligned}
& N_{1}=\left\langle\Sigma_{x x}\right\rangle-\left\langle\Sigma_{y y}\right\rangle, \\
& N_{2}=\left\langle\Sigma_{y y}\right\rangle-\left\langle\Sigma_{z z}\right\rangle,
\end{aligned}
$$

and the individual Brownian and hydrodynamic contributions for $\phi=0.45$ are shown in figures 10 and 11. Figures for the other volume fractions are available in Foss 


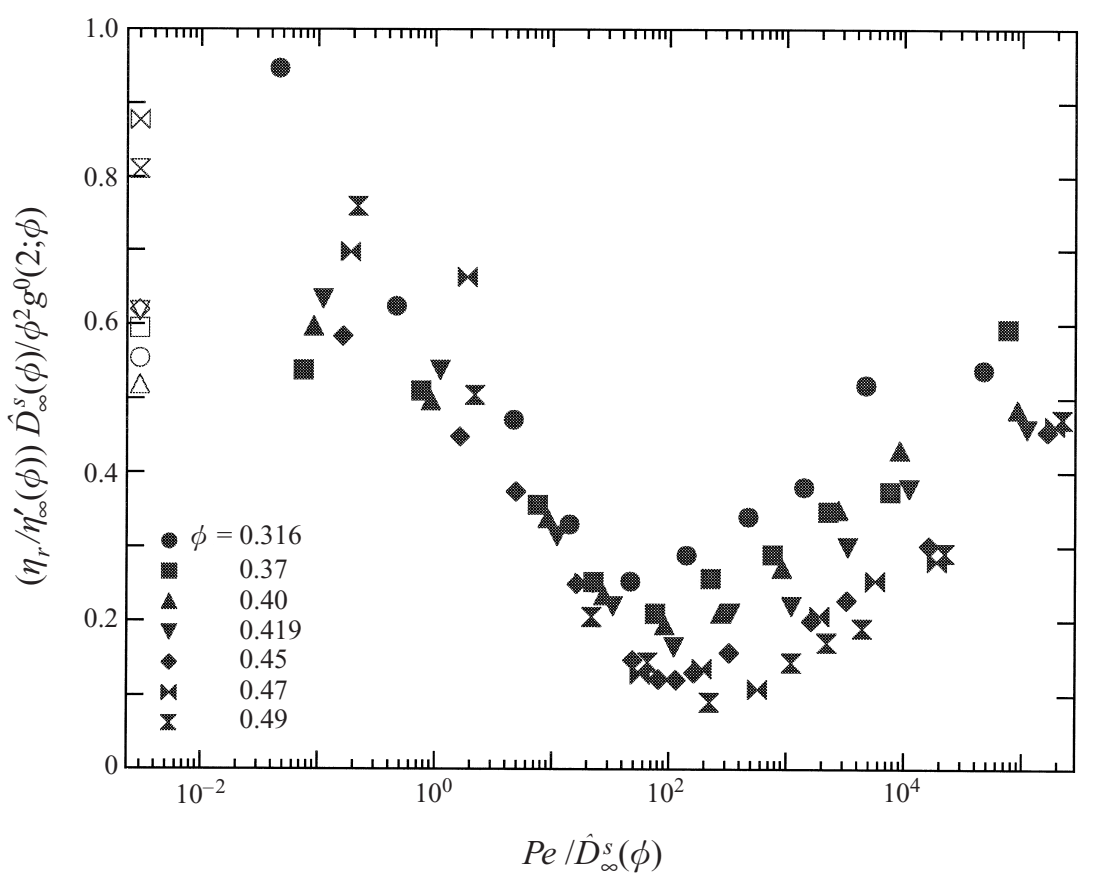

FiguRE 8. The difference of the total viscosity, $\eta_{r}$, from its equilibrium viscous contribution, $\eta_{\infty}^{\prime}$, scaled by $\phi^{2} g^{0}(2) / \hat{D}_{\infty}^{s}$ and plotted as a function of rescaled Péclet number $P e / \hat{D}_{\infty}^{s}$ for volume fractions $0.316 \leqslant \phi \leqslant 0.49$ with $N=27 . \hat{D}_{\infty}^{s}$ is the long-time self-diffusivity scaled by the Stokes-Einstein value $D=k T / 6 \pi \eta a$. The open symbols on the far left represent the $P e \rightarrow 0$ limits independently determined by an equilibrium Green-Kubo analysis.

(1999). Note that the hydrodynamic contributions are negative for all $\mathrm{Pe}$ while the Brownian contribution is positive for $N_{1}$ and negative for $N_{2}$.

Flow-reversal symmetry requires that both normal stress differences vanish as $P e \rightarrow 0$. At low Péclet numbers, the normal stress differences are dominated by the Brownian contributions resulting in a positive $N_{1}$ and a negative $N_{2}$ (Brady \& Vicic 1995). The quality of the data at low $P e$ in figures 10 and 11 is quite poor and one cannot discern a trend towards zero for small $P e$. It appears that the Brownian noise at low Péclet numbers that makes low-shear viscosities difficult to measure both in simulation and by experiment may be even worse for measuring normal stress differences. The normal stress differences show much greater fluctuation from one time step to the next, requiring very long runs and perhaps large systems to obtain meaningful averages. The signs of each of the normal stress differences, however, are discernible and correct.

The sign of the Brownian contribution to each normal stress difference can be ascertained by examining the microstructure and how it affects the integrand, $-\hat{\boldsymbol{r}} \hat{\boldsymbol{r}} \mathrm{g}(\boldsymbol{r})$, of the contact integral for the Brownian stress in (15). Figure 12 shows two projections of the radial distribution function, $g(\boldsymbol{r})$, onto the $(x, y)$-plane. The projections are divided into eight sections, each labelled with a plus or a minus. The signs correspond to the effect that a particle in that region would have on the normal stress differences given that the stress tensor is proportional to $-\hat{\boldsymbol{r}} \hat{\boldsymbol{r}} g(\boldsymbol{r})$. For determining the sign of the second normal stress difference, the microstructure is assumed to be axisymmetric outside the plane of shear as is seen below to be the case (cf. figure 21). The 


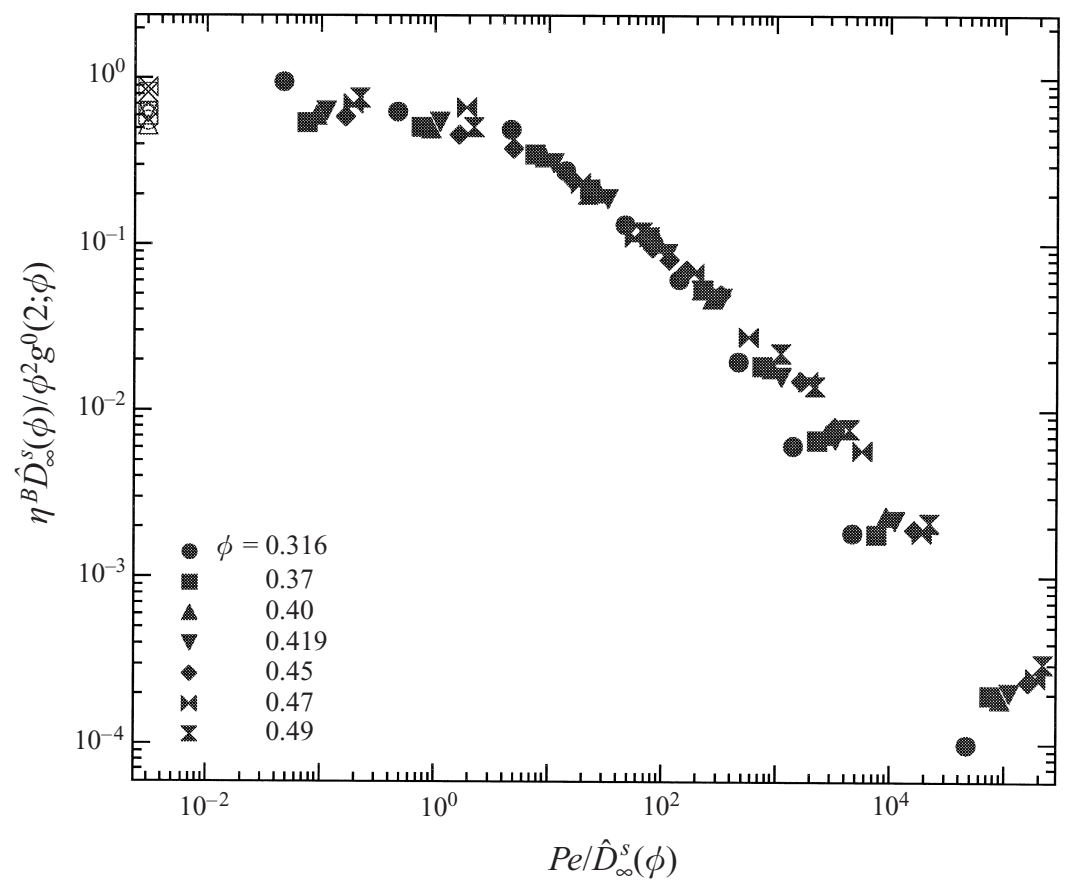

Figure 9. The Brownian contribution to the viscosity, $\eta^{B}$, scaled by $\phi^{2} g^{0}(2) / \hat{D}_{\infty}^{s}$ and plotted as a function of rescaled Péclet number $P e / \hat{D}_{\infty}^{s}$ for volume fractions $0.316 \leqslant \phi \leqslant 0.49$ with $N=27$. $\hat{D}_{\infty}^{s}$ is the long-time self-diffusivity scaled by the Stokes-Einstein value $D=k T / 6 \pi \eta a$. The open symbols on the far left represent the $P e \rightarrow 0$ limits independently determined by an equilibrium Green-Kubo analysis.

projections in figure 12 show that in addition to the buildup of particle probability in the compressional zone, some of the probability has been convected downstream into the neighbouring extensional zone creating an asymmetry about the compressional axes. From the relative magnitudes of the probabilities in the eight labelled sections of each projection, one can see that $N_{1}^{B}$ is positive and $N_{2}^{B}$ is negative due to the deficit of particle pairs along the extensional axes where Brownian motion pushes particles apart and hydrodynamic shear forces pull them apart. Physically, $N_{1}^{B}$ is positive because Brownian motion acts like a repulsive force between two particles and pushes them apart along the compressive and extensional axes, which would then push apart the plates of a rheometer.

As the Péclet number increases from zero, the deformation, and thus the Brownian contribution to the normal stress differences, also increases. A maximum is reached near $P e \approx 1$ as the deformation cannot keep up with the flow and the Brownian contributions decay at high Péclet numbers like 1/Pe (Brady \& Morris 1997), while the hydrodynamic contributions take over.

The signs of the hydrodynamic normal stress differences can also be explained by examining the suspension microstructure. At high Péclet number, the hydrodynamic stress is dominated by the boundary layer and the normal stress differences can be determined from (14) and thus the relevant tensor to examine is $\hat{\boldsymbol{r}} \hat{\boldsymbol{r}}(\hat{\boldsymbol{r}} \cdot \boldsymbol{E} \cdot \hat{\boldsymbol{r}}) g(\boldsymbol{r})$. Projections of the pair-distribution function onto the shear plane, similar to those used to explain the signs of the Brownian contribution, are shown in figure 13. Again, the projections are divided into eight sections, but this time with signs corresponding 


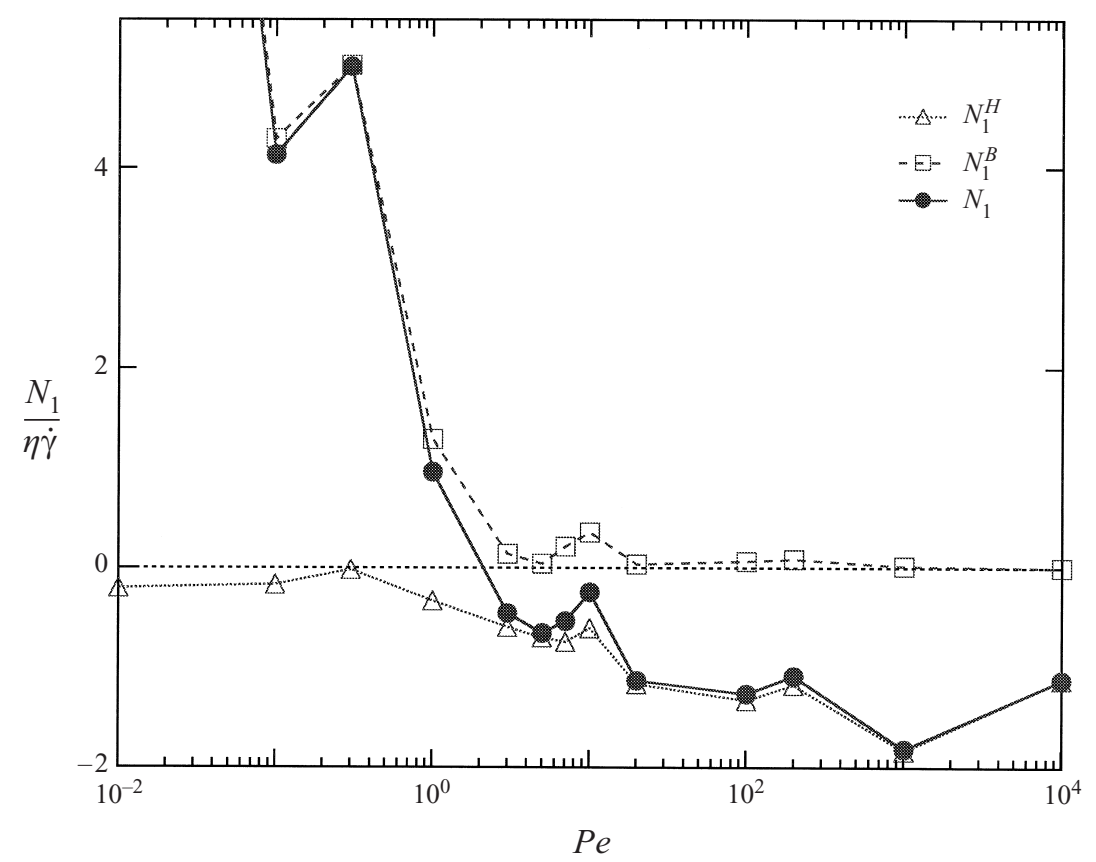

FiguRE 10. The hydrodynamic and Brownian contributions to the first normal stress difference for $N=27$ and $\phi=0.45$ as a function of the Péclet number.

to the contribution to the normal stress differences given by the stress tensor that is proportional to $\hat{\boldsymbol{r}} \hat{\boldsymbol{r}}(\hat{\boldsymbol{r}} \cdot \boldsymbol{E} \cdot \hat{\boldsymbol{r}})$. Examining the relative magnitudes of the probabilities in the eight labelled sections shows that both $N_{1}^{H}$ and $N_{2}^{H}$ are negative. As is the case at low Péclet numbers, the contributions from the two octants spanning the compressional axes essentially cancel, leaving the dominant contributions from the octants above the extensional axes. Here, the hydrodynamic stress is negative because the flow must pull apart the closely spaced particles stuck together by the lubrication forces. This pulling apart would in turn pull together the plates of a rheometer and hence give a negative first normal stress difference. The hydrodynamic contribution to the normal stress differences is much smaller than the Brownian at low $P e$ and increases in magnitude as the Péclet number is increased, resulting in a sign change of $N_{1}$ for $P e$ near 10.

In the pure hydrodynamic limit $\left(P e^{-1} \equiv 0\right)$ the normal stress differences are expected to vanish because of symmetry requirements. However, the singular boundarylayer behaviour as $P e \rightarrow \infty$ results in an asymmetric pair-distribution function at contact, clearly seen in figure 20 for $P e=10^{3}$, and normal stress differences that approach an $O(\eta \dot{\gamma})$ asymptote at very high Péclet numbers. Brady \& Morris (1997) show that for perfect hard spheres without interparticle forces, the asymmetry vanishes as $P e^{-0.22}$ as $P e \rightarrow \infty$. Again, hard spheres in the limit of pure hydrodynamics are singular. If a repulsive force is added, no matter how short-ranged, the asymmetry will not vanish as $P e \rightarrow \infty$, resulting in finite normal stress differences. As discussed earlier, it is numerically difficult to resolve the boundary layer at very high Péclet numbers and this difficulty may result in the particles behaving as if there were an interparticle force present and explain why the normal stress differences obtained from simulation do not vanish at high Péclet numbers. Experimentally, perfect hard spheres are difficult to achieve, and one should expect finite normal stress differences. 


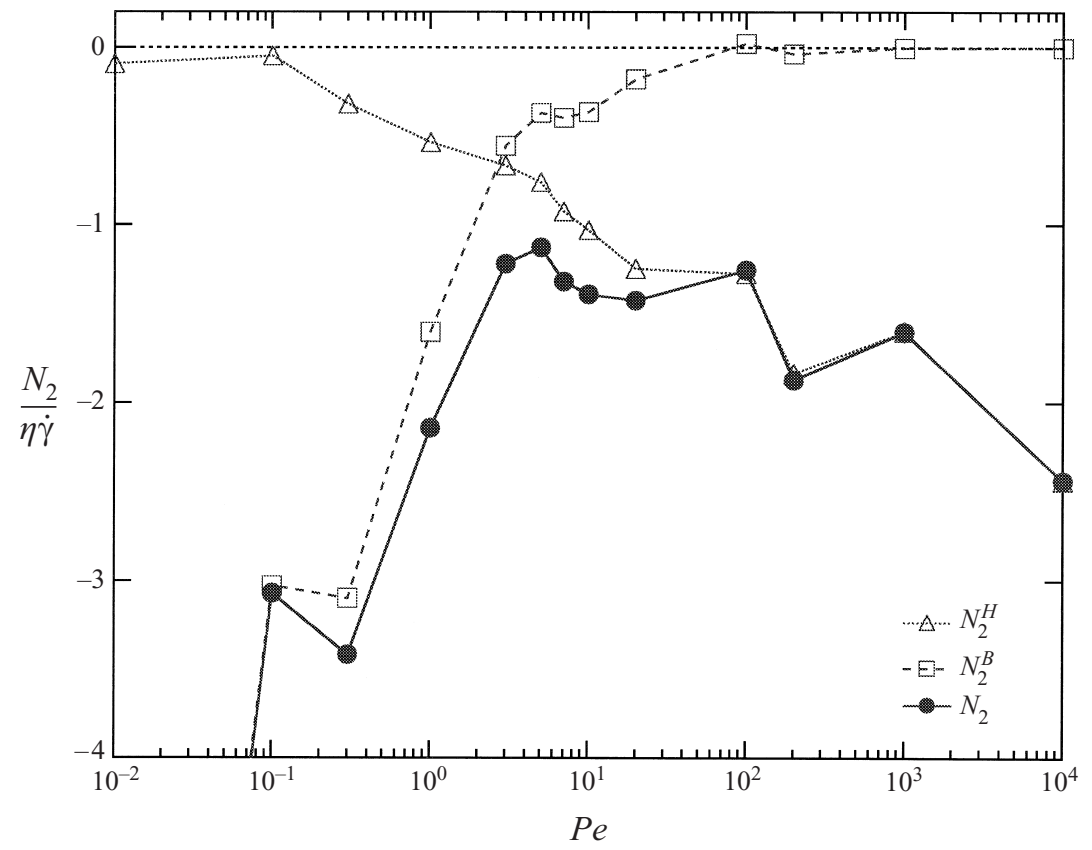

FIGURE 11. The hydrodynamic and Brownian contributions to the second normal stress difference for $N=27$ and $\phi=0.45$ as a function of the Péclet number.
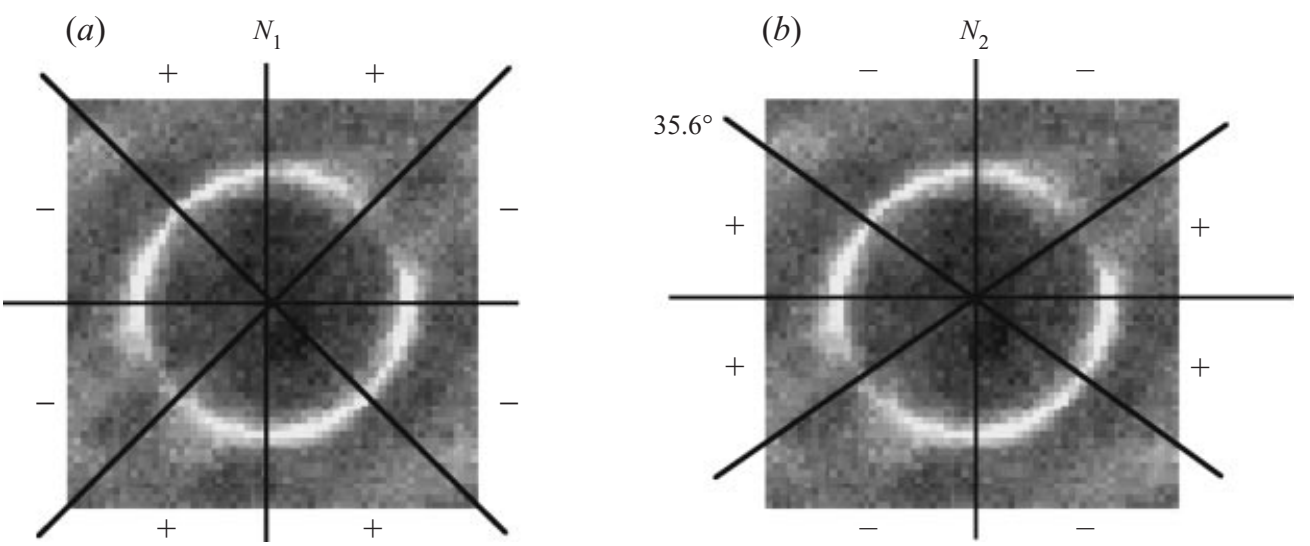

FIGURE 12. Projections of the pair distribution function, $g(\boldsymbol{r})$, into the $(x, y)$-plane for $N=27$, $\phi=0.45$, and $P e=1$. Light regions represent high probability and dark low. The projections are divided into eight sections each denoting the sign of the contribution to the Brownian normal stress difference from a particle in that region. The projections $(a)$ and $(b)$ are divided and labelled for determining $N_{1}$ and $N_{2}$, respectively. The Brownian stress is assumed to be proportional to $-\hat{r} \hat{r}$ and $g(\boldsymbol{r})$ is assumed to be symmetric about the $z$-axis.

Unfortunately, no experimental data are available for individual normal stress differences on model Brownian hard-sphere suspensions (or non-hard-sphere suspensions for that matter).

At high Péclet numbers the theory of Brady \& Morris (1997) used to collapse the shear-thickening viscosity can also be used to collapse the first and second normal stress differences. Figures 14 and 15 show $N_{1}$ and $N_{2}$ scaled with $\eta_{\infty}^{\prime}(\phi) \dot{\gamma} \phi^{2} g^{0}(2 ; \phi)$ as a 
(a)

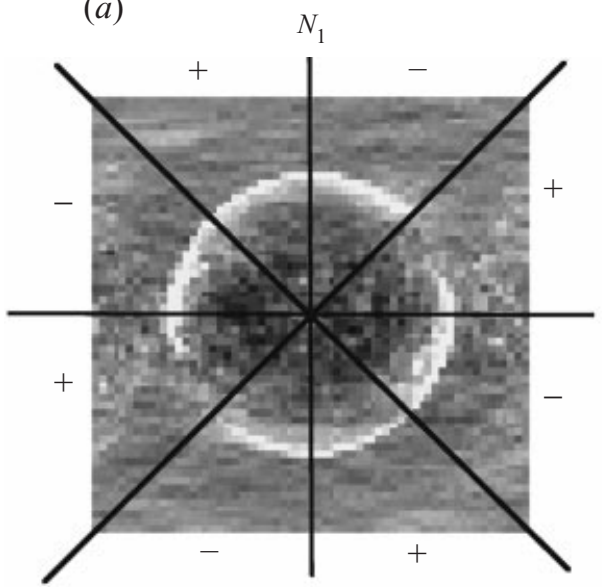

(b)

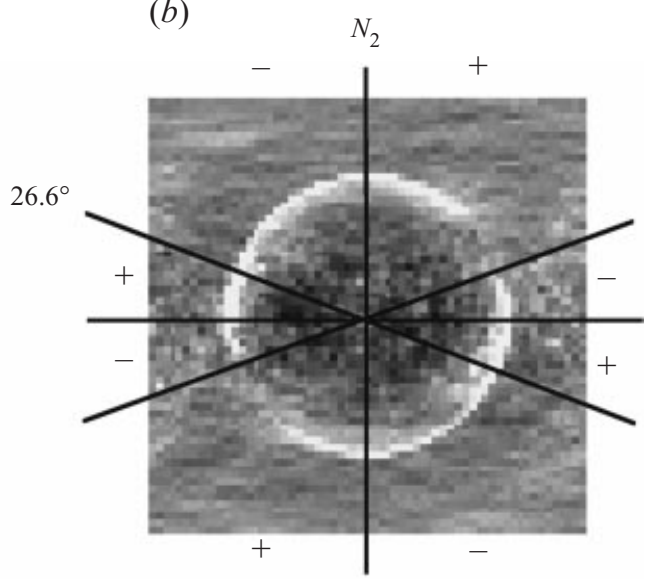

Figure 13. As figure 12 but for $P e=1000$. The Brownian stress is assumed to be proportional to $\hat{\boldsymbol{r}} \hat{\boldsymbol{r}}(\hat{\boldsymbol{r}} \cdot \boldsymbol{E} \cdot \hat{\boldsymbol{r}})$ and $g(\boldsymbol{r})$ is assumed to be symmetric about the $z$-axis.



FiguRE 14. The first normal stress difference, $N_{1}$, scaled by $\eta_{\infty}^{\prime} \dot{\gamma} \phi^{2} g^{0}(2)$ and plotted as a function of rescaled Péclet number $\overline{P e}=P e \eta_{\infty}^{\prime} / \eta$ for volume fractions $0.316 \leqslant \phi \leqslant 0.49$ with $N=27$.

function of the scaled Péclet number $\overline{P e}=P e \eta_{\infty}^{\prime}(\phi) / \eta$. The data for all concentration collapse reasonably well onto a single curve. The scaling theory with $\hat{D}(\phi)$ could also be used to scale the normal stress differences at low Péclet number, but the quality of the simulation data is too poor to draw any conclusions from the comparison.

The particle contribution to the stress defined in (9) is not traceless and the hydrodynamic functions necessary to compute the trace - the shear-rate-dependent osmotic pressure - were determined in Jeffrey, Morris \& Brady (1993) and have 


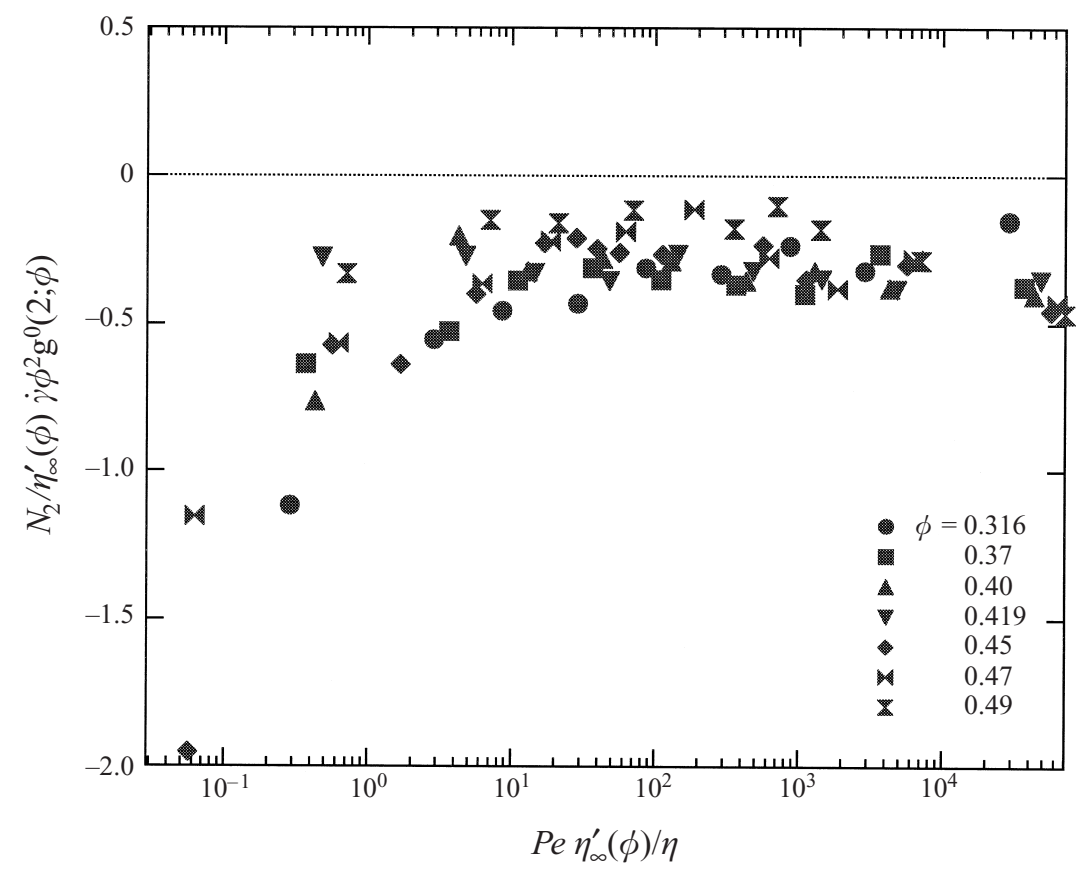

FIGURE 15 . The second normal stress difference, $N_{2}$, scaled by $\eta_{\infty}^{\prime} \dot{\gamma} \phi^{2} g^{0}(2)$ and plotted as a function of rescaled Péclet number $\overline{P e}=P e \eta_{\infty}^{\prime} / \eta$ for volume fractions $0.316 \leqslant \phi \leqslant 0.49$ with $N=27$.

been used in recent non-Brownian simulations (Yurkovetsky 1998). The extension to Brownian simulations has not yet been made and remains a goal for future work.

\subsection{Diffusion}

In figures 16 and 17 we show the average translational and rotational mobilities of a particle, $D_{0}^{s}$ and $D_{0}^{r}$, respectively, as a function of $P e$. At $P e \equiv 0$ these instantaneous mobilities (multiplied by $k T$ ) correspond to the short-time self-diffusion coefficients. The mobilities have been normalized by the infinite-dilution self-diffusion coefficients $k T / 6 \pi \eta a$ and $k T / 8 \pi \eta a^{3}$, respectively, and are averages of the separate $x x-, y y$ - and $z z$-components. The individual rotational mobilities are all identical to within the statistical uncertainty, while the $y y$ - and $z z$-components of the translation mobility are identical and the $x x$-component is perhaps $10 \%$ larger. The local mobility is to a very good approximation isotropic despite the structure formed during flow. The most important feature to note is that the mobilities remain roughly constant until the suspension starts to shear thicken, after which they decrease monotonically with increasing $P e$. This decrease is a manifestation of the closely touching particle clusters that form hindering the local motion of a particle.

The long-time mean-square displacement of a Brownian particle is convectively enhanced by the flow. For a simple shear flow the mean-square displacement is expected to grow in time according to (Elrick 1962; Morris \& Brady 1996)

$$
\begin{aligned}
\left\langle x^{2}(t)\right\rangle & =2 D_{x x} t+2 D_{y y} t\left[1+\frac{1}{3}(\text { Pe } t)^{2}\right], \\
\left\langle y^{2}(t)\right\rangle & =2 D_{y y} t, \\
\left\langle z^{2}(t)\right\rangle & =2 D_{z z} t, \\
\langle x(t) y(t)\rangle & =2 D_{x y} t+D_{y y} t(\text { Pe } t),
\end{aligned}
$$




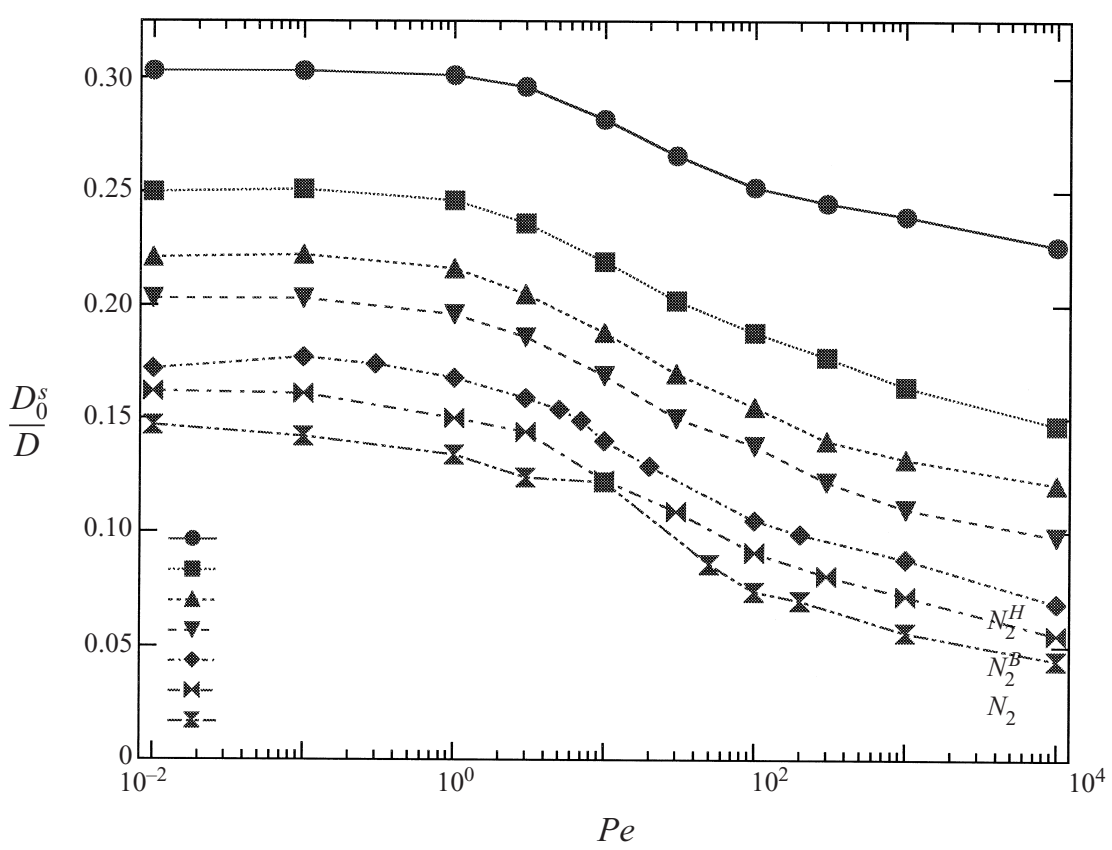

FIGURE 16. The average particle translational mobilities as a function of $P e$ for volume fractions $0.316 \leqslant \phi \leqslant 0.49$. At $P e \equiv 0$ these instantaneous mobilities (multiplied by $k T$ ) correspond to the short-time self-diffusion coefficients. The mobilities have been normalized by the diffusion coefficient of an isolated Brownian particle, $k T / 6 \pi \eta a$.

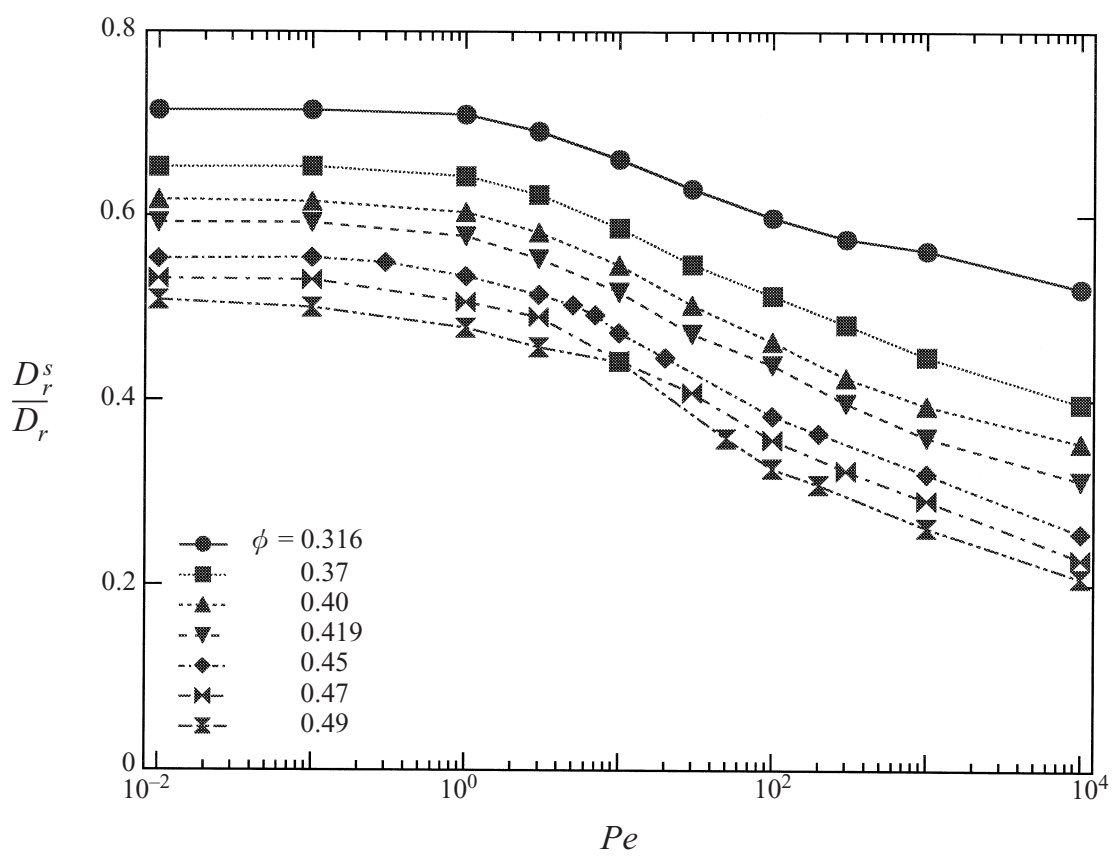

FIGURE 17. The average particle rotational mobilities as a function of $P e$ for volume fractions $0.316 \leqslant \phi \leqslant 0.49$. At $P e \equiv 0$ these instantaneous mobilities (multiplied by $k T$ ) correspond to the short-time self-diffusion coefficients. The mobilities have been normalized by the diffusion coefficient of an isolated Brownian particle, $k T / 8 \pi \eta a^{3}$. 
as $t \rightarrow \infty$. Because the $x$-displacement is dominated by the convective dispersion growing as $t^{3}$, it is not possible to determine the $D_{x x}$ and $D_{x y}$ coefficients by simply monitoring the displacements. An alternative method that removes the convectivelyenhanced dispersion and leaves the underlying diffusive motion is to subtract off the contributions to the particle motion in (4)-(5) due to the affine flow, $\Delta \boldsymbol{x}^{a}=$ $\langle\boldsymbol{U}\rangle \Delta t$, when calculating the mean-square displacements, which leads to the following temporal behaviour:

$$
\left\langle\left(\boldsymbol{x}-\boldsymbol{x}^{a}\right)\left(\boldsymbol{x}-\boldsymbol{x}^{a}\right)\right\rangle=2 \boldsymbol{D}_{\infty}^{s} t,
$$

as $t \rightarrow \infty$. All elements of the long-time self-diffusivity tensor, $\boldsymbol{D}_{\infty}^{s}$, can be calculated directly using this method. Apart from the $x y$-component, the other off-diagonal components of $\boldsymbol{D}_{\infty}^{s}$ were examined and found to be negligible. This method has been particularly useful in calculating the diffusivities in planar extensional flow (Sami 1996) where the affine flow has components in both the $x$ - and $y$-directions which lead to exponential rather than algebraic growth of the convectively enhanced terms.

We present results from steady-shear simulations of this work by examining the particle mean-square displacements for the small system at long times. Analysis done here at quite long times (between 10 and 20 dimensionless time units) would seem to be appropriate due to the infinite time limit in the definition of the long-time diffusivity (7); however, the mean-square displacements are time-correlation functions that relate particle positions from one time to another. Such correlation functions are quite difficult to accurately measure at long times due to the growth of statistical noise with time. In a related work (Foss \& Brady 1999) we use a different method that focuses on the behaviour of the mean-square displacements during many short simulations, but still long enough to have attained the long-time asymptote, which we believe produces more accurate and consistent data. We include the long-time analysis in this work despite the increased noise in the data because it is at long times and serves as a good comparison with our other work. The statistical error in the long-time self-diffusivities is about $30 \%$. For a more detailed discussion of the long-time self-diffusivity in flow, see Foss \& Brady (1999).

The Péclet-number dependence for $\phi=0.45$ of the $x x-, y y$-, $z z$-components of $\boldsymbol{D}_{\infty}^{s}$ normalized by the diffusion coefficient of an isolated particle is shown in figure 18 . Data for the other volume fractions can be found in Foss (1999). As $P e \rightarrow 0$ the diffusivities approach the long-time self-diffusivities of equilibrium dispersions and agree well with the experimental measurements of van Megen, Underwood \& Snook (1986); a comparison of these data with experiment can be found in Brady (1994). The action of the flow is to enhance the self-diffusivity, with the leading correction scaling as $P e^{3 / 2}$ (Morris \& Brady 1996). As the Péclet number increases, the self-diffusivities continue to increase without the 'dip' at intermediate Péclet numbers that was present in the previous work (Phung et al. 1996). The 'dip' was evidence of the formation of an ordered phase in this region of Péclet number causing the diffusivities to drop. The monotonically increasing behaviour of the diffusivities is an indication that no ordered phase is present in the current simulations (cf. figure 20). As $P e \rightarrow \infty$, the results show that the normalized self-diffusivities grow approximately linearly with $P e$, or in dimensional terms $D_{y y} \sim \dot{\gamma} a^{2}$, corresponding to hydrodynamic diffusion. The simulation results for the shear-induced or hydrodynamic diffusivities show quite a bit of scatter, but are in reasonable agreement (within a factor of 2; see Foss \& Brady 1999 ) with the experiments of Eckstein, Bailey \& Shapiro (1977), Leighton \& Acrivos (1987) and Phan \& Leighton (1993). Again, the singular effect of Brownian motion is important in leading to diffusive behaviour as $P e \rightarrow \infty$. A pure hydrodynamic 


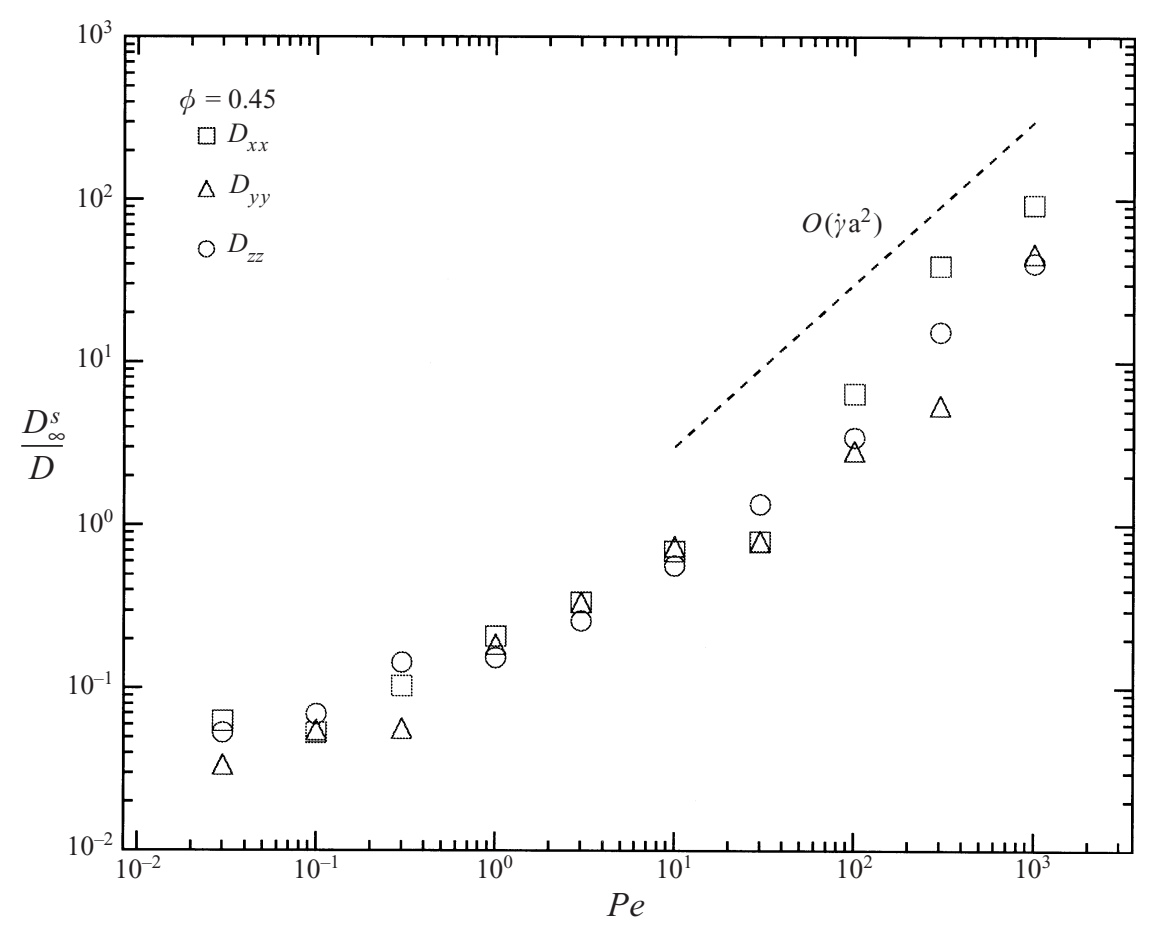

FIGURE 18 . The long-time self-diffusivities in the velocity, $D_{x x}$, velocity-gradient, $D_{y y}$, and vorticity, $D_{z z}$, directions for $\phi=0.45$ as a function of $P e$. The diffusivities have been normalized by the diffusion coefficient of an isolated Brownian particle, $k T / 6 \pi \eta a$. At large $P e$ the dimensional long-time diffusivities scale hydrodynamically as $\dot{\gamma} a^{2}$.

system is completely deterministic and, although the evolution equations for particle positions are highly nonlinear and may give rise to deterministic chaos, the small amount of Brownian motion (or surface roughness or interparticle forces) provides the necessary irreversibility for loss of dependence on initial conditions. We were not able to determine long-time self-diffusivities from the simulations with $N>27$ as the runs were not long enough to reach the asymptotic temporal behaviour; thus, we do not know the effect of the size of the simulation cell on the diffusivities. The values obtained here are in agreement with the monolayer results of Bossis \& Brady (1987) when allowance is made for converting area fraction to volume fraction.

The enhancement of the long-time self-diffusivity by flow is indicative of a different mechanism for creating diffusive motion in sheared suspensions. Direct particleparticle 'collisions' induced by the shearing motion are responsible for the random walk. The action of this mechanism is seen in figure 19 where the long-time selfdiffusivities in the $z z$-direction at $P e=0.01$ and $P e=1000$ are shown as a function of the volume fraction. At low Péclet numbers the long-time diffusivity decreases with increasing concentration, while at high Péclet number it increases with increasing $\phi$. At low $P e$ particle interactions hinder the motion of a tagged particle, while at high $P e$ they help. At intermediate Péclet numbers one should therefore find the long-time diffusivities to be independent of, or non-monotonic in, $\phi$. Unfortunately, experimental data are only available at zero and infinite $P e$. 


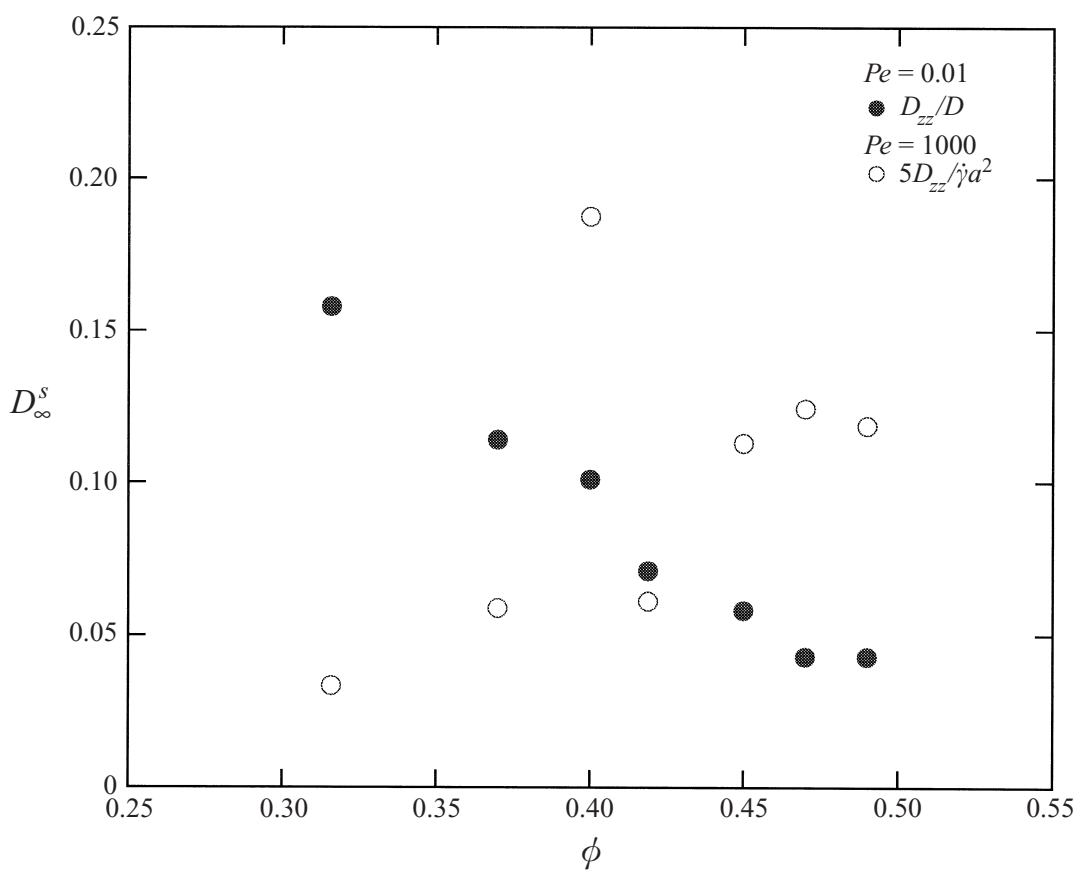

FIGURE 19. The $z z$-component of the long-time self-diffusivity tensor as a function of $\phi$ at both high and low Péclet numbers. The diffusivity is non-dimensionalized by the Stokes-Einstein value $D=k T / 6 \pi \eta a$ for $P e=0.01$ and by $\frac{1}{5} \dot{\gamma} a^{2}$ for $P e=1000$.

\subsection{Structure}

An analysis of the microstructure shows that no ordered phase is present for the full range of $P e$ and volume fractions studied, $0.316 \leqslant \phi \leqslant 0.49$. Figure 20 shows the projection of the pair-distribution function onto the plane of shear for $\phi=0.45$. Near equilibrium, the flow provides a weak perturbation to the equilibrium behaviour. The hint of an eight-fold symmetry seen in figures 20 and 21 at small $P e$ is due to the small size of the simulation cell. This structure is completely absent for larger systems as seen in figure 22. As the Péclet number increases, the first nearest-neighbour peak at contact becomes intensified along the compressive axes of the flow and becomes less intense along the extensional axes. Physically, particles are being convected together along the compressive axes (the upstream side), come into near contact, rotate together as a doublet, and then depart on the downstream side where Brownian motion and the shearing flow act together to separate the particles. The intensification and sharpening of the nearest-neighbour peak as the Péclet number increases is further qualitative evidence of the $O\left(P e^{-1}\right)$ boundary layer discussed by Brady \& Morris (1997).

It is important to note that in contrast to the earlier work (Phung \& Brady 1992; Phung 1993; Phung et al. 1996), no ordered structure is obtained using the correct high- $P e$ evolution equation (5). Figure 21 shows the projection of the pair-distribution function in the velocity-gradient-vorticity plane and no hexagonal pattern typical of flow-induced string-ordering is evident. Analysis of larger systems $(N=123)$ has also failed to show any order (see figure 22). The ordering in the earlier work can be directly attributed to the $\nabla \cdot \boldsymbol{R}_{F U}^{-1}$ term in the evolution equation which acts like a radially repulsive Brownian force (Bossis \& Brady 1987). In the earlier work, this term is 'enhanced' by a factor of $P e^{1 / 2}$ in comparison to the other Brownian displacement 


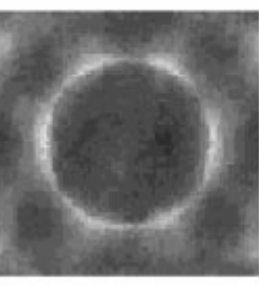

$P e=0.01$

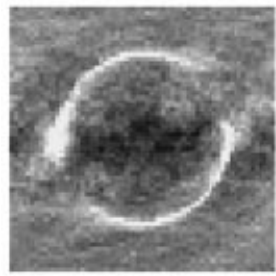

$P e=10$

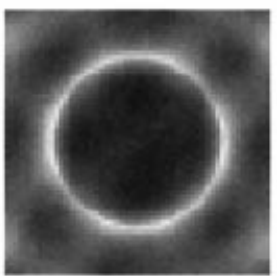

0.1

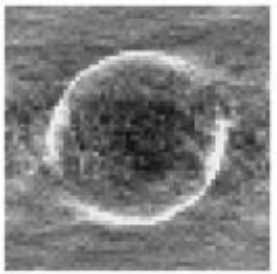

20

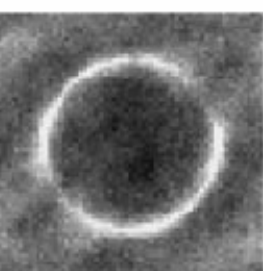

1

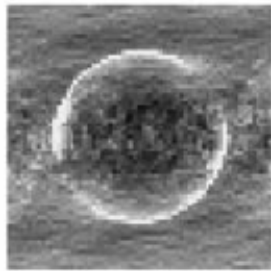

100
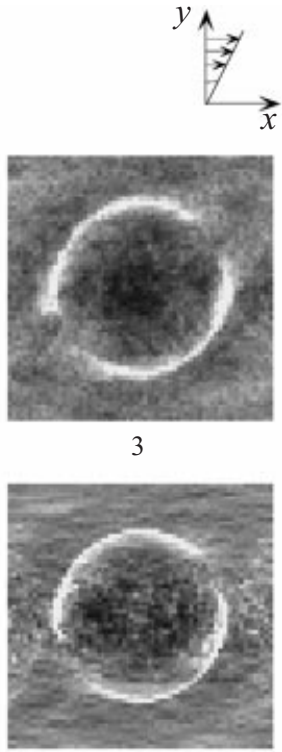

1000

FigURE 20. The pair-distribution function projected onto the velocity-velocity-gradient plane for $N=27$ and $\phi=0.45$.

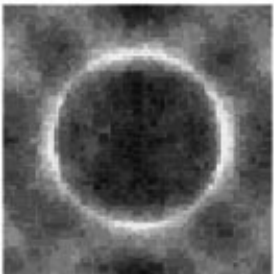

$P e=0.01$



$P e=10$

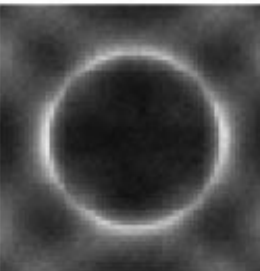

0.1

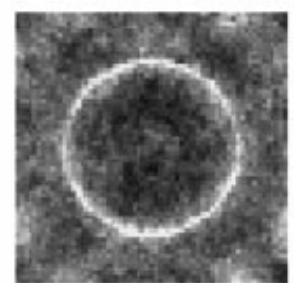

20



1



100


3

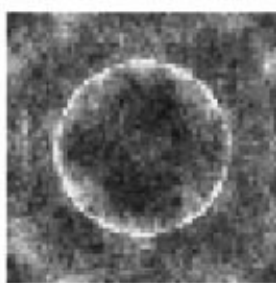

1000

FIGURE 21. The pair-distribution function projected onto the velocity-gradient-vorticity plane for $N=27$ and $\phi=0.45$.

term, providing an additional repulsion between particles. This repulsion prevents the particles from getting close enough for the singular hydrodynamic lubrication forces to come into play. These close-ranged forces cause particles to form temporary doublets that rotate in shear flow and disrupt any order that may form. A simulation 

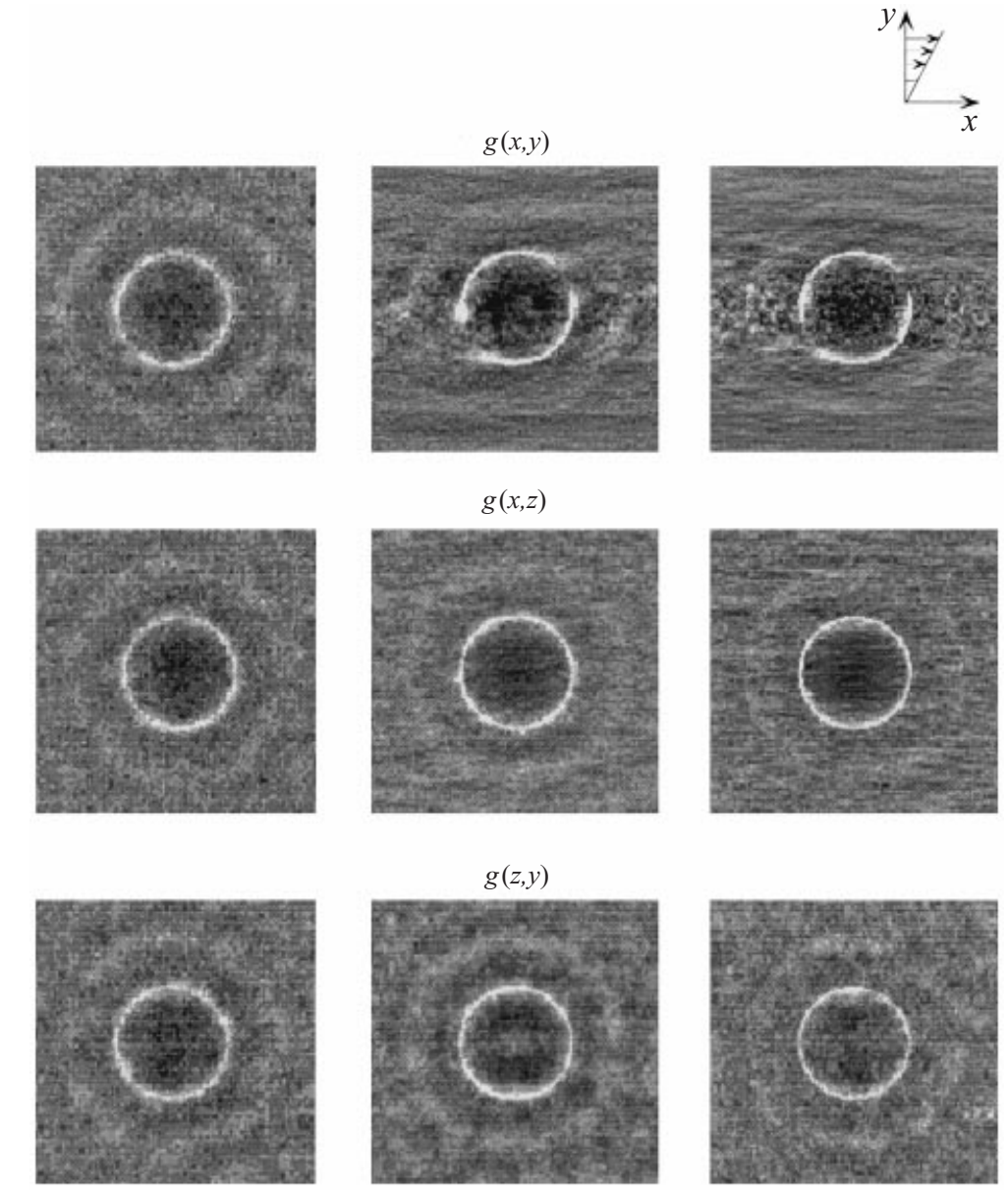

$$
P e=0.43
$$

$$
P e=10
$$

$$
P e=1000
$$

FIGURE 22. Projections of the pair-distribution function in all three planes for $N=123$ at $P e=0.43,10$, and 1000 .

performed with a pairwise-additive short-range repulsive DLVO-type interparticle force also removes the lubrication forces and enables the suspension to order (see figure 23). The form for the DLVO-type force is for two constant-charge spheres immersed in an ionic solvent (Russel, Saville \& Schowalter 1989), which is the same form used in previous Stokesian Dynamics simulations (Bossis \& Brady 1984; Yurkovetsky 1998), and is given by

$$
\boldsymbol{F}^{P}=-\left|\boldsymbol{F}^{P}\right| \frac{\mathrm{e}^{-\tau h}}{1-\mathrm{e}^{-\tau h}} \hat{\boldsymbol{r}}
$$

where, $\hat{\boldsymbol{r}}$ is the unit vector along the line of centres of a pair of particles, $h=(r-2 a) / a$ is non-dimensional particle separation, and $\tau=\kappa a$ is the non-dimensional inverse Debye length ( $\kappa^{-1}$ is the Debye length). For this particular run, we use $\left|\boldsymbol{F}^{P}\right|=200 k T / a$, and $\tau=200$.

Flow-induced ordering is commonly seen experimentally in electrostaticallystabilized dispersions (Ackerson 1990; Laun et al. 1992; Chen, Ackerson \& Zukoski 1994) which utilize DLVO-type repulsive forces between the particles to prevent 
(a)



(c)



(b)



(d)



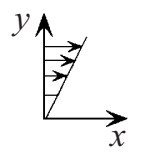

FIGURE 23. Pair distribution functions for $N=27, \phi=0.40$ and $P e=100$ for the hard-sphere case $(a, b)$ shows no flow-induced ordering while using a DLVO-type repulsive force $(c, d)\left(\dot{\gamma}^{*}=0.5\right.$, $\tau=100$ ) shows hexagonally-packed string ordering. The pair-distribution function is projected onto the velocity-velocity-gradient plane for $(a, c)$ and the velocity-gradient-vorticity plane for $(b, d)$.

flocculation. Evidently, these repulsive forces are sufficiently long-ranged and strong enough to exclude the lubrication forces and allow the string-ordered phase to form in certain ranges of shear rate. Our first paper on Stokesian Dynamics (Bossis \& Brady 1984) showed order for a suspension of non-Brownian particles interacting through hydrodynamic and repulsive colloidal forces. Non-equilibrium molecular dynamics simulations have also exhibited string formation (Erpenbeck 1984; Hess 1985; Heyes 1986), as have Brownian Dynamics simulations (Heyes 1988; Xue \& Grest 1990; Rastogi 1995; Foss \& Brady 2000) where all hydrodynamic interactions are neglected, i.e. setting $\boldsymbol{R}_{F U}=\boldsymbol{I}$, and $\boldsymbol{R}_{F E}=\boldsymbol{R}_{S U}=\boldsymbol{R}_{S E}=0$ in (2)-(10c). What the above examples all have in common is the absence of short-ranged lubrication forces that tend to disrupt any order that may form. Interestingly, sterically stabilized colloidal dispersions in which short polymer chains are grafted onto particle surfaces have not been observed to string order (Bender \& Wagner 1996). Evidently, the steric layers do not prevent the lubrication interactions.

There has been no evidence of any effect of system size for the simulations performed for $\phi<0.49$. At $\phi=0.49$ and $P e=100$, some order was found for $N=27$, while a simulation with $N=63$ shows no order (see figure 24); no noticeable change in the viscosities was observed, however. These finite-size effects become increasingly important as the volume fraction is increased. $N=27$ simulations for $\phi>0.49$ have shown a strong tendency to order over the full range of Péclet numbers despite the lack of order in the limited number of runs we have been able to perform for larger systems where $N=123$ and, unlike the case above, the effect of the order on the viscosities was quite significant. Clearly, larger runs must be used to examine the rheological behaviour for these very dense suspensions.

The absence of string ordering for non-repulsive systems makes a strong argument about the connection between shear thinning/thickening and flow-induced ordering. 
(a)



(c)

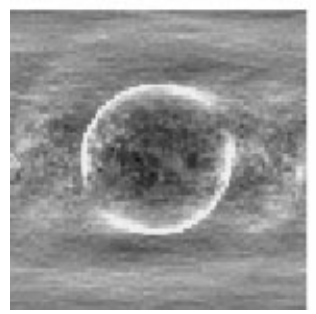

(b)



(d)

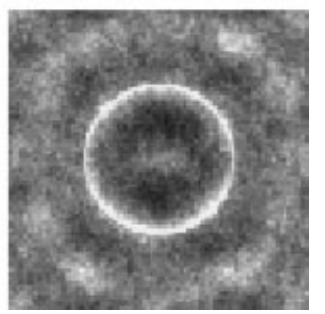



FIGURE 24. Pair distribution functions for $\phi=0.49$ and $P e=100$ using $N=27(a, b)$ shows hexagonally-packed string ordering while increasing the system size to $N=63(c, d)$, shows no flow-induced ordering. The pair-distribution function is projected onto the velocity-velocity-gradient plane for $(a, c)$ and the velocity-gradient-vorticity plane for $(b, d)$.

It has often been stated that shear thinning is caused by the formation of an ordered phase causing the suspension to flow more easily. Similarly, the onset of shear thickening has often been connected with the melting of this order. Since no order has been observed here, there is no necessary relationship between flow-induced order and shear thinning/thickening.

\section{Conclusions}

In this paper we have investigated the non-equilibrium behaviour of concentrated colloidal dispersions of hard spheres in simple shear flow by Stokesian Dynamics simulation. The suspension is governed by the competition between Brownian and hydrodynamic forces as measured by the Péclet number. At low Péclet number the equilibrium structure is perturbed but the suspension shear thins. This shear thinning results from the decrease of the direct Brownian contribution to the stress as the structural deformation cannot keep up with the shear flow. The hydrodynamic contribution to the viscosity remains constant and equal to the high-frequency dynamic viscosity throughout the shear-thinning process.

At high Péclet numbers $(P e>10)$, the effects of Brownian motion give way to hydrodynamic forces which result in a thin boundary layer of high particle probability near contact whose thickness scales as $O\left(P e^{-1}\right)$ where hydrodynamic and Brownian forces balance (as shown by Brady \& Morris 1997). More particle pairs near contact increase the effects of lubrication forces causing the viscosity to increase. As the Péclet number is increased, the boundary layer becomes thinner and the probability density in the boundary layer increases, further enhancing the effects of lubrication, and the suspension shear thickens.

In the infinite-Péclet-number limit a suspension of perfect hard spheres appears to be singular in that a small amount of Brownian motion or interparticle forces has a 
dramatic effect on structure and properties. The thin $O\left(P e^{-1}\right)$ boundary layer leads to an asymmetry in the pair-distribution function and a loss of flow reversal symmetry. This asymmetry produces $O(\eta \dot{\gamma})$ hydrodynamic normal stress differences and $O\left(\dot{\gamma} a^{2}\right)$ shear-induced particle diffusivities in the infinite- $P e$ limit.

No flow-induced ordering was observed over the range of volume fractions studied here $(0.316 \leqslant \phi \leqslant 0.49)$ due to the action of close-ranged lubrication forces. Systems with repulsive forces are known to exhibit this type of ordering due to the exclusion of hydrodynamic lubrication. Otherwise, analogous behaviour in terms of shear thinning and thickening and structure formation has been seen in colloidal dispersions with repulsive forces. Indeed, one can often scale the behaviour of repulsive systems by using an equivalent volume fraction that incorporates the short-range repulsion into an effective particle radius (Ackerson 1990; Brady 1993b). Long-range repulsive forces are somewhat different in that hydrodynamic interactions are minimized or absent, which results in a different scaling for the dependence of the viscosity on concentration (Brady 1993b), but the shear thinning and ordering phenomena are still present.

Although the results we have presented here are for small system sizes, the viscosities appear to agree quantitatively with experiment. There are no measurements of normal stresses or diffusivities as a function of $P e$, however. The results give confidence that Stokesian Dynamics can be used to quantitatively study the behaviour of colloidal dispersions over a wide range of conditions.

The highest volume fraction studied in this work is $\phi=0.49$. It is well known that monodisperse hard spheres undergo an equilibrium phase transition at $\phi \approx 0.494$ to a crystalline structure. Suspensions above this transition exhibit shear-induced melting upon inception of simple shear flow. It is possible that hard-sphere suspensions at these large densities may also exhibit a string-ordered microstructure at high shear rates. Simulations of such dense suspensions require larger system sizes than are currently practical using conventional Stokesian Dynamics. As simulation algorithms improve and hardware computational speed increases, we look forward to studying the behaviour of very dense colloidal dispersions.

This work would not have been possible without the assistance of Thanh N. Phung who wrote the Stokesian Dynamics codes. The work was supported in part by grants CTS-9020646, CTS-9420415, and INT-9415673 from the National Science Foundation.

\section{REFERENCES}

ACKERSON, B. J. 1990 Shear induced order and shear processing of model hard sphere suspensions. J. Rheol. 34, 553

Ball, R. C. \& Melrose, J. R. 1995 Lubrication breakdown in hydrodynamic simulations of concentrated colloids. Adv. Colloid Interface Sci. 59, 19.

Banchio, A. J., Bergenholtz, J. \& NäGele, G. 1999 Rheology and dynamics of colloidal suspensions. Phys. Rev. Lett. 82, 1792.

BATCHELOR, G. K. 1977 The effect of Brownian motion on the bulk stress in a suspension of spherical particles. J. Fluid Mech. 83, 97.

Bender, J. W. \& Wagner, N. J. 1995 Optical measurement of the contribution of colloidal forces to the rheology of concentrated suspensions. J. Colloid Interface Sci. 172, 171.

BENDER, J. W. \& WAGNER, N. J. 1996 Reversible shear thickening in monodisperse and bidisperse colloidal dispersions. J. Rheol. 40, 899.

Bossis, G. \& Brady, J. F. 1984 Dynamic simulation of sheared suspensions. I. General method. J. Chem. Phys. 80, 5141. 
Bossis, G. \& Brady, J. F. 1987 Self-diffusion of Brownian particles in concentrated suspensions under shear. J. Chem. Phys. 87, 5437.

Bossis, G. \& Brady, J. F. 1989 The rheology of Brownian suspensions. J. Chem. Phys. 91, 1866.

Bossis, G., Brady, J. F. \& Mathis, C. 1988 Shear-induced structure in colloidal suspensions I: Numerical simulation. J. Colloid Interface Sci. 126, 1.

Bossis, G., Meunier, A. \& Brady, J. F. 1991 Hydrodynamic stress of fractal aggregates of spheres. J. Chem. Phys. 94, 5064.

Brady, J. F. 1993 a Brownian motion, hydrodynamics, and the osmotic pressure. J. Chem. Phys. 98, 3335.

Brady, J. F. $1993 b$ The rheological behaviour of concentrated colloidal dispersions. J. Chem. Phys. 99, 567.

Brady, J. F. 1994 The long-time self-diffusivity in concentrated colloidal dispersions. J. Fluid Mech. 272, 109.

BRady, J. F. \& Bossis, G. 1985 The rheology of concentrated suspensions of spheres in simple shear flow by numerical simulation. J. Fluid Mech. 155, 105.

Brady, J. F. \& Bossis, G. 1988 Stokesian dynamics. Ann. Rev. Fluid Mech. 20, 111.

Brady, J. F., Lester, J., Phillips, R. \& Bossis, G. 1988 Dynamic simulation of hydrodynamically interacting suspensions. J. Fluid Mech. 195, 257.

Brady, J. F. \& MorRis, J. F. 1997 Microstructure of strongly-sheared suspensions and its impact on rheology and diffusion. J. Fluid Mech. 348, 103.

Brady, J. F. \& Vicic, M. 1995 Normal stresses in colloidal dispersions. J. Rheol. 39, 545.

Chen, L. B., Ackerson, B. J. \& Zukoski, C. F. 1994 Rheological consequences of microstructural transitions in colloidal crystals. J. Rheol. 38, 193.

D’Haene, P., Mewis, J. \& Fuller, G. G. 1993 Scattering dichroism measurements of flow-induced structure of a shear thickening suspension. J. Colloid Interface Sci. 156, 350.

Dratler, D. I. \& Schowalter, W. R. 1996 Dynamic Simulation of suspensions of non-Brownian hard spheres. J. Fluid Mech. 325, 53.

Durlofsky, L. J., Brady, J. F. \& Bossis, G. 1995 Dynamic simulation of hydrodynamically interacting particles. J. Fluid Mech. 180, 21.

Eckstein, E. C., Bailey, D. G. \& Shapiro, A. H. 1977 Self-diffusion in shear flow of a suspensions. J. Fluid Mech. 79, 191.

Elrick, D. E. 1962 Source functions for diffusion in uniform shear flow. Austral. J. Phys. 15, 283.

ERPENBECK, J. J. 1984 Shear viscosity of the hard-sphere fluid via nonequilibrium molecular dynamics. Phys. Rev. Lett. 52, 1333.

Foss, D. R. 1999 Rheological behaviour of colloidal suspensions: the effects of hydrodynamic interactions. PhD Thesis, California Institute of Technology.

Foss, D. R. \& Brady, J. F. 1999 Self-diffusion in sheared suspensions by dynamic simulation. J. Fluid Mech. 401, 243.

Foss, D. R. \& Brady, J. F. 2000 Brownian Dynamics simulation of hard-sphere colloidal dispersions. J. Rheol. (to appear).

Hess, S. 1985 Shear-induced melting and reentrant positional ordering in a system of spherical particles. Intl J. Thermophys. 6, 657.

HeYEs, D. M. 1986 Some physical consequences of large shear rates on simple fluids. J. Chem. Phys. 85, 997.

HeYes, D. M. 1988 Shear thinning of dense suspensions modeled by Brownian dynamics. Phys. Lett. 132, 399.

Jeffrey, D. J., Morris, J. F. \& Brady, J. F. 1993 The pressure moments for two rigid spheres in low-Reynolds-number flow. Phys. Fluids A 5, 2317.

Kruif, C. G. De, Iersel, E. M. F. van, VriJ, A. \& Russel, W. B. 1985 Hard sphere colloidal dispersion: viscosity as a function of shear rate and volume fraction. J. Chem. Phys. 83, 4717.

Laun, H. M., Bung, R., Hess, S., Loose, W., Hess, O., Hahn, K., Hädicke, E., Hingmann, R., SCHMidT, F. \& Linder, P. 1992 Rheological and small angle neutron scattering investigation of shear-induced particle structures of concentrated polymer dispersions submitted to plane Poiseuille and Couette flow. J. Rheol. 36, 743.

Leighton, D. \& ACrivos, A. 1987 Measurement of self-diffusion in concentrated suspensions of spheres. J. Fluid Mech. 177, 109. 
Lionberger, R. A. 1998 Shear thinning of colloidal dispersions. J. Rheol. 42, 843.

MACKAY, M. E. \& KAFFASHI, B. 1995 Stress jumps of colloidal suspensions, measurements of the elastic-like and viscous-like stress components. J. Colloid Interface Sci. 174, 117.

Megen, W. van, Underwood, S. M. \& SNOOK, I. 1986 Tracer diffusion in concentrated colloidal dispersions. J. Chem. Phys. 85, 4065.

Melrose, J. R. \& Ball, R. C. 1995 The pathological behaviour of sheared hard spheres with hydrodynamic interactions. Europhys. Letters 32, 535.

Morris, J. F. \& Brady, J. F. 1996 Self diffusion in sheared suspensions. J. Fluid Mech. 312, 223.

NäGele, G. \& Bergenholtz, J. 1998 Linear viscoelasticity of colloidal mixtures. J. Chem. Phys. 108, 9893.

Phan, S. E. \& Leighton, D. L. 1993 Measurement of the shear-induced tracer diffusivity in concentrated suspensions. J. Fluid Mech. (submitted).

Phung, T. N. 1993 Behavior of concentrated colloidal suspensions by Stokesian Dynamics simulation. PhD Thesis, California Institute of Technology.

Phung, T. N. \& Brady, J. F. 1992 Microstructured fluids: structure, diffusion and rheology of colloidal dispersions. In Slow Dynamics in Condensed Matter (ed. Kawasaki, Tokuyama \& Kawakatsu). AIP Conference Proc. 256, p. 391. Am. Inst. of Phys. New York.

Phung, T. N., Brady, J. F. \& Bossis, G. 1996 Stokesian Dynamics simulations of Brownian suspensions. J. Fluid Mech. 313, 181.

Pusey, P. N., Segrè, P. N., Behrend, O. P., Meeker, S. P. \& Poon, W. C. K. 1997 Dynamics of concentrated colloidal suspensions. Physica A 235, 1.

Rastogi, S. R. 1995 Nonequilibrium Brownian dynamics of colloidal suspensions. PhD Thesis, University of Delaware.

Russel, W. B., Saville, D. A. \& Schowalter, W. R. 1989 Colloidal Dispersions. Cambridge University Press.

SAMI, S. 1996 Stokesian Dynamics simulations of Brownian suspensions in extensional flow. MS Thesis, California Institute of Technology.

Segrè, P. H., Meeker, S. P., Pusey, P. N. \& Poon, W. C. K. 1995 Viscosity and structural relaxation in suspensions of hard-sphere colloids. Phys. Rev. Lett. 75, 958.

Shikata, T. \& Pearson, D. S. 1994 Viscoelastic behaviour of concentrated spherical suspensions. J. Rheol. 38, 601.

Thomas, D. G. 1965 Transport characteristics of suspensions. VIII. A note on the viscosity of Newtonian suspensions of uniform spherical particles. J. Colloid Interface Sci. 20, 267.

Verberg, R., Schepper, I. M. DE \& Cohen, E. G. D. 1997 Viscosity of colloidal suspensions. Phys. Rev. E 55, 3143.

VICIC, M. 1999 Rheology and microstructure of complex fluids: dispersions, emulsions and polymer solutions. PhD Thesis, California Institute of Technology.

WerfF, J. C. van deR \& Kruif, C. G. DE 1989 Hard-sphere colloidal dispersions: the scaling of rheological properties with particle size, volume fraction, and shear rate. J. Rheol. 33, 421.

Werff, J. C. van der, Kruif, C. G. De, Blom, C. \& Mellema, J. 1989 Linear viscoelastic behaviour of dense hard-sphere dispersions. Phys. Rev. A 39, 67.

Xue, W. \& Grest, G. S. 1990 Shear-induced alignment of colloidal particles in the presence of a shear flow. Phys. Rev. Lett. 64, 419.

YURKOVETSKY, Y. 1998 I. Statistical mechanics of bubbly liquids. II. Behavior of sheared suspensions of non-Brownian particles. PhD Thesis, California Institute of Technology. 